\title{
Invariom-model refinement and Hirshfeld surface analysis of well-ordered solvent-free dibenzo-21-crown-7
}

\section{Dennis Wiedemann and Julia Kohl}

Acta Cryst. (2017). C73, 654-659

\section{IUCr Journals CRYSTALLOGRAPHY JOURNALS ONLINE}

Copyright (C) International Union of Crystallography

Author(s) of this paper may load this reprint on their own web site or institutional repository provided that this cover page is retained. Republication of this article or its storage in electronic databases other than as specified above is not permitted without prior permission in writing from the IUCr.

For further information see http://journals.iucr.org/services/authorrights.html 


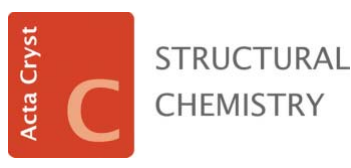

ISSN 2053-2296

Received 13 July 2017

Accepted 27 July 2017

Edited by A. L. Spek, Utrecht University, The Netherlands

Keywords: crown ethers; crystal structure; invariom refinement; intermolecular interactions; Hirshfeld surface; interaction energies.

CCDC references: $1565414 ; 1565413$

Supporting information: this article has supporting information at journals.iucr.org/c

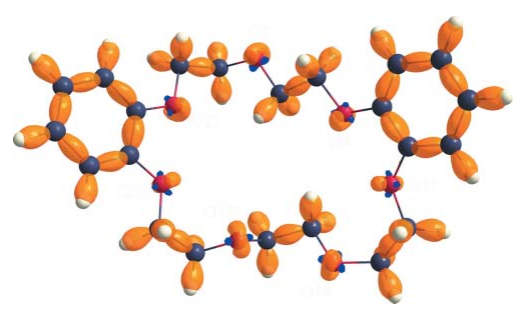

C 2017 International Union of Crystallography

\section{Invariom-model refinement and Hirshfeld surface analysis of well-ordered solvent-free dibenzo-21- crown-7}

\author{
Dennis Wiedemann* and Julia Kohl
}

Institut für Chemie, Technische Universität Berlin, Strasse des 17 Juni 135, 10623 Berlin, Germany. *Correspondence e-mail: dennis.wiedemann@chem.tu-berlin.de

Crown ethers and their supramolecular derivatives are well-known chelators and scavengers for a variety of cations, most notably heavier alkali and alkalineearth ions. Although they are widely used in synthetic chemistry, available crystal structures of uncoordinated and solvent-free crown ethers regularly suffer from disorder. In this study, we present the X-ray crystal structure analysis of well-ordered solvent-free crystals of dibenzo-21-crown-7 (systematic name: dibenzo[ $b, k]-1,4,7,10,13,16,19$-heptaoxacycloheneicosa-2,11-diene, $\mathrm{C}_{22} \mathrm{H}_{28} \mathrm{O}_{7}$ ). Because of the quality of the crystal and diffraction data, we have chosen invarioms, in addition to standard independent spherical atoms, for modelling and briefly discuss the different refinement results. The electrostatic potential, which is directly deducible from the invariom model, and the Hirshfeld surface are analysed and complemented with interaction-energy computations to characterize intermolecular contacts. The boat-like molecules stack along the $a$ axis and are arranged as dimers of chains, which assemble as rows to form a three-dimensional structure. Dispersive $\mathrm{C}-\mathrm{H} \cdots \mathrm{H}-\mathrm{C}$ and $\mathrm{C}-\mathrm{H} \cdots \pi$ interactions dominate, but nonclassical hydrogen bonds are present and reflect the overall rather weak electrostatic influence. A fingerprint plot of the Hirshfeld surface summarizes and visualizes the intermolecular interactions. The insight gained into the crystal structure of dibenzo-21-crown-7 not only demonstrates the power of invariom refinement, Hirshfeld surface analysis and interactionenergy computation, but also hints at favourable conditions for crystallizing solvent-free crown ethers.

\section{Introduction}

Macrocyclic crown ethers are established chelators particularly suited for substituted ammonium ions and heavy-metal cations (Pedersen, 1967). The title compound, dibenzo-21crown-7 (systematic name: dibenzo[b,k]-1,4,7,10,13,16,19heptaoxacycloheneicosa-2,11-diene, also 6,7,9,10,12,13,20,21,23,24-decahydrodibenzo[ $b, k][1,4,7,10,13,16,19]$ heptaoxacycloheneicosin; Chemical Abstracts Service, 2017), (I), is known to selectively form stable complexes with ions of heavy alkali and alkaline-earth metals, most notably caesium (Blasius \& Nilles, 1984).

Although Petersen had synthesized the molecule in the late 1960s, the first reliable crystal structure of the nitromethane adduct of (I) was not established until the mid-1990s (Burns et al., 1996). Only three closely related derivatives, namely dimethyldibenzo-21-crown-7 (Owen \& Nowell, 1978), 4,4'-ditert-octyldibenzo-21-crown-7 (Sachleben et al., 1997) and tetranitrodibenzo-21-crown-7 (Mäkelä et al., 2016), have been characterized using X-ray diffraction, probably because of the notorious tendency of crown ethers to disorder, especially when uncoordinated. 
Table 1

Experimental details.

Chemical formula $=\mathrm{C}_{22} \mathrm{H}_{28} \mathrm{O}_{7}, M_{\mathrm{r}}=404.44$, crystal system $=$ monoclinic, space group $=P 2_{1} / c$ and $Z=4$. The experiment was carried out at $150.00(10) \mathrm{K}$ with $\mathrm{Cu} K \alpha$ radiation using an Agilent SuperNova (single source) diffractometer. Absorption was corrected for using numerical methods based on Gaussian integration over a 14-faceted crystal model (grid dimensions: $10^{3}$, beam-profile correction for $0.18 \times 0.18 \mathrm{~mm}^{2}$ ). An empirical absorption correction was then performed using spherical harmonics and frame scaling (minimum factor: 0.662; maximum factor: 1.465), as implemented in CrysAlis PRO (Rigaku Oxford Diffraction, 2015).

\begin{tabular}{|c|c|c|}
\hline & IAM & Invariom model \\
\hline \multicolumn{3}{|l|}{ Crystal data } \\
\hline$a, b, c(\AA)$ & \multicolumn{2}{|c|}{$4.9801(1), 17.4771(2), 23.1000(2)$} \\
\hline$\beta\left({ }^{\circ}\right)$ & \multicolumn{2}{|c|}{$94.124(1)$} \\
\hline$V\left(\AA^{6}\right)$ & \multicolumn{2}{|c|}{$2005.37(5)$} \\
\hline$\mu\left(\mathrm{mm}^{-1}\right)$ & \multicolumn{2}{|c|}{0.82} \\
\hline Crystal size (mm) & \multicolumn{2}{|c|}{$0.74 \times 0.14 \times 0.08$} \\
\hline \multicolumn{3}{|l|}{ Data collection } \\
\hline$T_{\min }, T_{\max }$ & \multicolumn{2}{|c|}{$0.598,1.000$} \\
\hline $\begin{array}{l}\text { No. of measured, independent and observed } \\
\quad[I>2 \sigma(I)] \text { reflections }\end{array}$ & \multicolumn{2}{|c|}{$13236,3925,3559$} \\
\hline$R_{\text {int }}$ & \multicolumn{2}{|c|}{0.018} \\
\hline$(\sin \theta / \lambda)_{\max }\left(\AA^{-1}\right)$ & \multicolumn{2}{|c|}{0.622} \\
\hline \multicolumn{3}{|l|}{ Refinement } \\
\hline$R\left[F^{2}>2 \sigma\left(F^{2}\right)\right], w R\left(F^{2}\right), S$ & $0.038,0.103,1.05$ & $0.030,0.084,0.98$ \\
\hline No. of reflections & 3925 & 3664 \\
\hline No. of parameters & 262 & 290 \\
\hline $\mathrm{H}$-atom treatment & $\mathrm{H}$-atom parameters constrained & Only H-atom displacement parameters refined \\
\hline Weighting scheme & $\begin{array}{l}w=1 /\left[\sigma^{2}\left(F_{\mathrm{o}}^{2}\right)+(0.0497 P)^{2}+0.7337 P\right] \\
\quad \text { where } P=\left(F_{\mathrm{o}}^{2}+2 F_{\mathrm{c}}^{2}\right) / 3\end{array}$ & $\begin{array}{l}w=1 /\left[\sigma^{2}\left(F_{\mathrm{o}}^{2}\right)+(0.0497 P)^{2}+0.1 P\right] \\
\quad \text { where } P=\left(F_{\mathrm{o}}^{2}+2 F_{\mathrm{c}}^{2}\right) / 3\end{array}$ \\
\hline$\Delta \rho_{\max }, \Delta \rho_{\min }$, r.m.s. $\Delta \rho\left(\mathrm{e} \AA^{-3}\right)$ & $0.39,-0.28,0.04$ & $0.41,-0.24,0.03$ \\
\hline
\end{tabular}

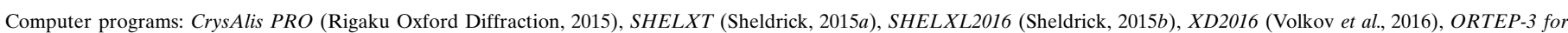
Windows (Farrugia, 2012), Mercury (Macrae et al., 2008), OLEX2 (Dolomanov et al., 2009) and WinXD (Volkov et al., 2016).

In this article, we present the crystal structure of wellordered solvent-free dibenzo-21-crown-7. Inspired by a talk given by K. Woźniak (Sanjuan-Szklarz et al., 2016) and because of the suitability of our data set, we go beyond the

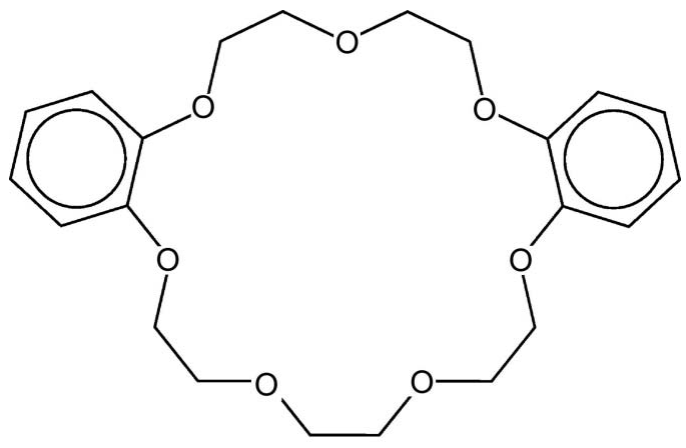

(I)

spherical independent atom model (IAM), which is the standard in crystal structure modelling. For the purpose of full characterization, we also apply the invariom approach, which assigns an aspherical atomic form factor to each atom according to its nature and chemical environment (Dittrich et al., 2004).

\section{Experimental}

\subsection{Crystallization}

Dibenzo-21-crown-7 (98\%) and toluene were purchased from Acros Organics. The crown ether was dried in vacuo overnight. The solvent was distilled over sodium benzophenone ketyl and stored over molecular sieves ( $4 \AA$ ) before use.

In a Schlenk tube, a sample of (I) was dissolved in toluene $(5 \mathrm{ml})$. The Schlenk tube was then connected to a second empty such tube via a curved glass connector. The solution was frozen by immersing the first tube in liquid nitrogen, and the whole apparatus was evacuated to $c a 10^{-2} \mathrm{hPa}$. The frozen solution was then allowed to warm to room temperature ( $c a$ $293 \mathrm{~K}$ ) and the empty Schlenk tube was dipped into a Dewar vessel containing slightly cooler water ( $c a$ 291 K). The small temperature gradient allowed the toluene to evaporate slowly from the solution, resulting in the growth of colourless single crystals suitable for diffractometry within five days. We were satisfied with obtaining crystals under these special conditions and did not further optimize the crystal size with respect to suitability for the micro-focus source.

\subsection{Refinement}

Crystal data, data collection and structure refinement details are summarized in Table 1. Firstly, a conventional IAM refinement was performed using SHELXL2016 (Sheldrick, 2015b) with OLEX2 (Dolomanov et al., 2009) as a front end. All $\mathrm{H}$ atoms were located in difference Fourier maps and constrained using a standard riding model, with $\mathrm{C}-\mathrm{H}=$ $0.99 \AA$ for methylene groups and $0.95 \AA$ for aromatic groups, and with $U_{\text {iso }}(\mathrm{H})=1.2 U_{\text {eq }}(\mathrm{C})$.

Based on the refined IAM, MoleCoolQt (Hübschle \& Dittrich, 2011) was used to assign and check invarioms and 


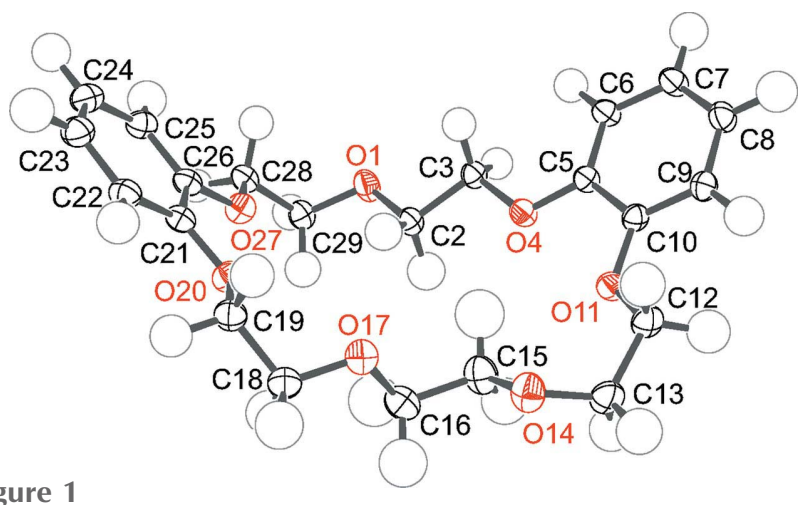

Figure 1

An ORTEP representation, with displacement ellipsoids and spheres drawn at the $50 \%$ probability level, of the molecular structure of (I) according to the invariom model (colour key: $\mathrm{C}$ atoms black, $\mathrm{O}$ atoms red and $\mathrm{H}$ atoms grey).

local coordinate systems, import them from the generalized invariom database (Dittrich et al., 2013) and set up input files for the next step (cf. Table S1 in the supporting information). The resulting model, which is based on multipoles according to the Hansen-Coppens formalism (Hansen \& Coppens, 1978), was then refined with $X D 2016$ (Volkov et al., 2016) against a data set merged using tools implemented in Win GX (Farrugia, 2012). To assure comparability of weighted results, the limit for observed reflections was lowered to $I>2 \sigma(I)$ and a SHELXL-like weighting scheme with $a(X D)=p(S H E L X L)$ and $b(X D)=0.1$ was applied, so that the weighted goodnessof-fit $S$ converged to a value near unity.

$\mathrm{H}$ atoms were placed at database-derived optimized distances from the carrier atom, which were reset after every refinement cycle, and refined with isotropic displacement parameters. To correctly assess the hydrogen bonds in the invariom model, bonding distances and angles were calculated using the module $X D G E O M$ of $X D 2016$. The result of the invariom refinement is shown in Fig. 1. The displacement ellipsoid plot for the IAM refinement is nearly identical.

\subsection{Further computations}

The electrostatic potential was directly derived from the invariom model using XD2016 (Volkov et al., 2016) and projected on the Hirshfeld surface using MoleCoolQt (Hübschle \& Dittrich, 2011). The Tonto routines (Jayatilaka \& Grimwood, 2003) implemented in CrystalExplorer (Turner et al., 2017) were used to calculate Hirshfeld surfaces and fingerprint plots, as well as to compute scaled interaction energies via density-functional theory (DFT) with the hybrid functional B3LYP and Pople's polarized split-valence basis set 6-31G(d,p) (Mackenzie et al., 2017).

\section{Results and discussion}

\subsection{Invariom versus IAM refinement}

The number of reflections used in both refinements deviate from each other because of algorithmic differences: SHELXL, the de-facto standard in conventional IAM refinements, adjusts against intensity data and includes all reflections in the final refinement steps (negative intensities below a modifiable threshold are set to the threshold value), whereas XD2016 (Volkov et al., 2016), the standard in charge-density studies, excludes the unobserved reflections. For the calculation of structure factors and $R$ values, however, all reflections are included in both cases.

Under these preconditions, the invariom model outperformed the IAM in terms of $R$ factors, especially $w R\left(F^{2}\right)$ for all reflections, and the r.m.s. of the difference electron-density $\Delta \rho$. Whereas the IAM exhibits local maxima at lone-pair positions, on delocalized $\pi$ and also many $\sigma$ bonds, the invarioms model bond and lone-pair electron density at the expense of core electron density. This is manifested in the deformation electron density $\Delta \rho_{\text {def }}=\rho($ invariom $)-\rho($ IAM $)$ (Fig. 2) clearly showing these features. As the results of the invariom refinement are superior to those of the IAM refinement, all the following considerations are based on the former.

\subsection{Molecular geometry}

All bond lengths within the molecule of (I) are in the expected range. The ethylene groups adopt - at least approximately - staggered conformations. Bonds to adjacent $\mathrm{O}$ atoms are oriented gauche, with the exception of the $\mathrm{O} 1-$ $\mathrm{C} 2-\mathrm{C} 3-\mathrm{O} 4$ fragment, which shows an anti conformation. This results in an overall boat-like shape of the molecule, which is governed by two planes: one containing the chain segment C18-C29 (r.m.s. deviation $=0.0370 \AA$ ), roughly coinciding with the crystallographic plane (124), and one containing atoms $\mathrm{O} 1-\mathrm{C} 13$ and $\mathrm{C} 29$ (r.m.s. deviation = $0.1035 \AA$ ) , roughly coinciding with $(\overline{125})$. The planes intersect at an angle of $76.377(1)^{\circ}$. Atoms O14-O17 are part of neither plane ( $c f$. Fig. S1 in the supporting information).

Interestingly, a comparison of the average displacement parameters for the shorter $\left[\bar{U}_{\text {eq }}(\mathrm{C} / \mathrm{O})=0.029(4) \AA^{2}\right.$ and $\left.\bar{U}_{\text {iso }}(\mathrm{H})=0.048(6) \AA^{2}\right]$ and the longer chain $\left[\bar{U}_{\text {eq }}(\mathrm{C} / \mathrm{O})=\right.$ $0.036(4) \AA^{2}$ and $\left.\bar{U}_{\text {iso }}(\mathrm{H})=0.068(6) \AA^{2}\right]$ connecting the aromatic rings also hints at slightly less order in the latter. The maximum residual electron density is also found in its envir-

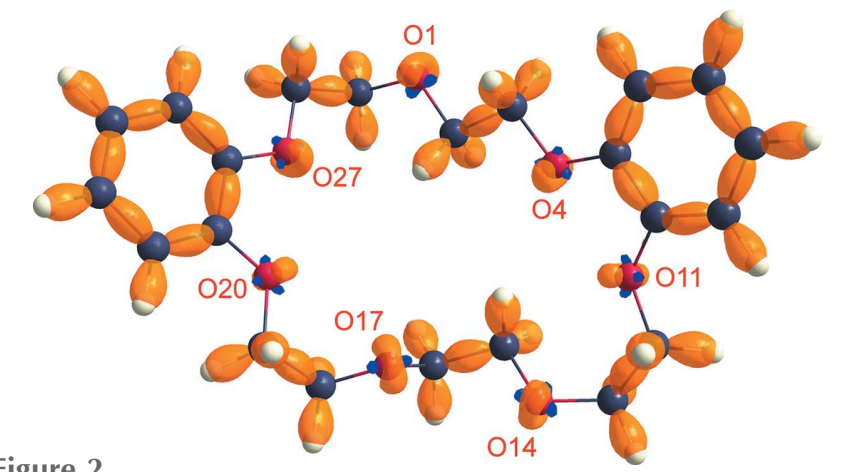

Figure 2

Isosurfaces of the deformation electron density $\Delta \rho_{\text {def }}= \pm 0.3 \mathrm{e} \AA^{-3}$ (positive is orange and negative is blue). The molecule is shown in a balland-stick representation with arbitrary radii (colour key: $\mathrm{C}$ atoms black, $\mathrm{O}$ atoms red and $\mathrm{H}$ atoms white). 


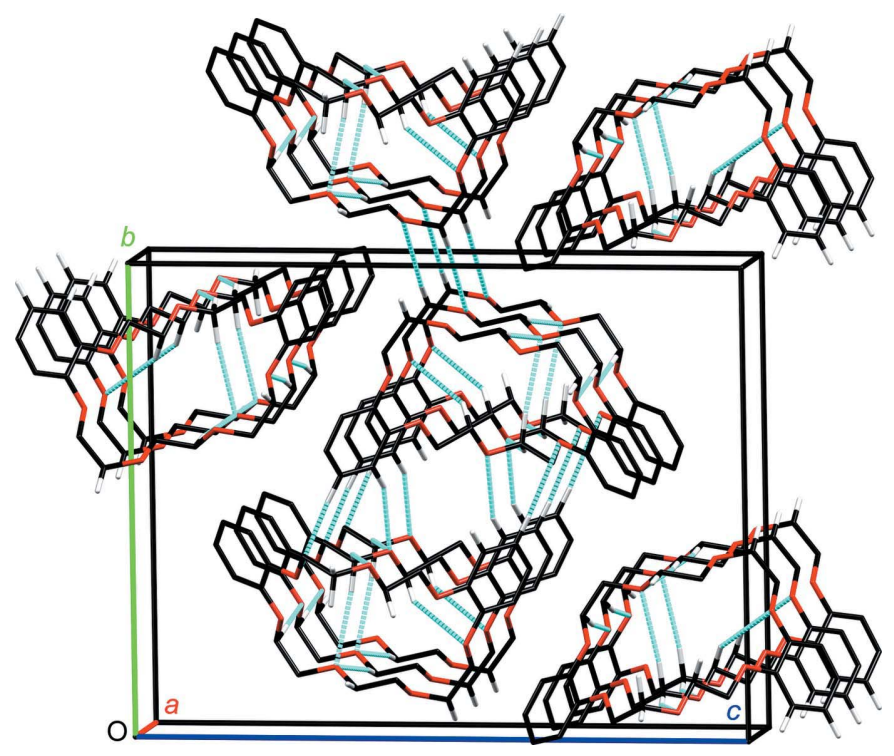

Figure 3

The packing diagram of (I) in the crystal, shown in a capped-stick representation with arbitrary radius (colour key: black $\mathrm{C}$, red $\mathrm{O}$ and white $\mathrm{H}$ atoms, and blue dashed lines NCHBs). $\mathrm{H}$ atoms not involved in the hydrogen bonding and dangling hydrogen bonds have been omitted for clarity.

onment. The small increase when switching from the IAM to the invariom model ( $c f$. Table 1 ) is due to the now adequate modelling of bond and lone-pair electron density; the effect of minor disorder becomes more pronounced. However, the high degree of order in the crystals remains exceptional for uncoordinated crown ethers.

\subsection{Intermolecular interactions}

3.3.1. Contacts and packing. Two kinds of intermolecular interactions govern the crystal packing, i.e. dispersive contacts and nonclassical hydrogen bonds (NCHBs) between $\mathrm{C}-\mathrm{H}$ groups and $\mathrm{O}$ atoms. Among the dispersive contacts, various side-on interactions between aromatic rings and catechol $\mathrm{CH}_{2}$ groups, as well as edge-on contacts between the out-of-plane part of the longer chain and the $\mathrm{C} 21-\mathrm{C} 26$ ring, are predominant.

To assess the hydrogen-bond network, we defined NCHBs using relatively strict criteria: $\mathrm{C} \cdots \mathrm{O} \leq 4.5 \AA$ and $\mathrm{C}-\mathrm{H} \cdots \mathrm{O} \geq$ $150^{\circ}$ (Desiraju \& Steiner, 1999). Inspection of the twofold hydrogen-bonded $\mathrm{H} 2 \mathrm{~A}$ atom led to the rejection of the longer smaller-angle bond to atom O14 so that eight intermolecular NCHBs are considered to be present (see Table 2). Based on their C . O distance, seven of these may be grouped into one of two classes (3.80-4.10 and 4.25-4.45 $\AA$ ). One bond is particularly short at 3.5631 (13) .

In the crystal, the boat-like molecules are stacked to build infinite chains along $a$, donating four and accepting one NCHB on the 'inner side' - and vice versa on the 'outer side' (Fig. 3). Three belong to the class of shorter NHCBs and two to the class of longer NHCBs. This correlates well with the results of computation: with a total energy of $-88.8 \mathrm{~kJ} \mathrm{~mol}^{-1}$
Table 2

Hydrogen-bond geometry $\left(\AA,^{\circ}\right)$ for Invariom.

\begin{tabular}{lllll}
\hline$D-\mathrm{H} \cdots A$ & $D-\mathrm{H}$ & $\mathrm{H} \cdots A$ & $D \cdots A$ & $D-\mathrm{H} \cdots A$ \\
\hline $\mathrm{C} 2-\mathrm{H} 2 A \cdots \mathrm{O} 11^{\mathrm{i}}$ & 1.10 & 2.85 & $3.8410(14)$ & 151 \\
$\mathrm{C} 6-\mathrm{H} 6 \cdots \mathrm{O} 1^{\mathrm{ii}}$ & 1.08 & 2.57 & $3.5631(13)$ & 153 \\
$\mathrm{C} 7-\mathrm{H} 7 \cdots \mathrm{O} 27^{\mathrm{iii}}$ & 1.08 & 3.23 & $4.2104(14)$ & 151 \\
$\mathrm{C} 13-\mathrm{H} 13 B \cdots \mathrm{O} 14^{\mathrm{iv}}$ & 1.10 & 3.26 & $4.3405(15)$ & 170 \\
$\mathrm{C} 16-\mathrm{H} 16 A \cdots \mathrm{O} 17^{\mathrm{i}}$ & 1.10 & 3.21 & $4.2956(16)$ & 172 \\
$\mathrm{C} 19-\mathrm{H} 19 B \cdots \mathrm{O} 20^{\mathrm{v}}$ & 1.10 & 3.01 & $4.0769(14)$ & 164 \\
$\mathrm{C} 29-\mathrm{H} 29 A \cdots \mathrm{O} 17^{\mathrm{i}}$ & 1.10 & 2.85 & $3.9393(15)$ & 172 \\
$\mathrm{C} 29-\mathrm{H} 29 B \cdots \mathrm{O} 1^{\mathrm{i}}$ & 1.10 & 3.42 & $4.4459(14)$ & 156
\end{tabular}

Symmetry codes: (i) $x+1, y, z$; (ii) $-x+2,-y+1,-z+1$; (iii) $-x+1,-y+1$, $-z+1$; (iv) $-x,-y+2,-z+1$; (v) $x-1, y, z$.

per molecule ( $c f$. Table S2 and Fig. S1 in the supporting information), the interactions in these chains are by far the strongest in the crystal. The shortest hydrogen bonds connect the chains to dimers, in which each molecule donates and accepts one NCHB of this type to/from the same neighbour related by a centre of inversion (Fig. 3, centre of the unit cell). In keeping with these hydrogen bonds being the shortest, one finds the second lowest electrostatic and total interaction energy $\left(-29.1 \mathrm{~kJ} \mathrm{~mol}^{-1}\right)$ within the dimers. The remaining two longer NCHBs connect the chain dimers to form infinite rows (Fig. 3, upper cell boundary). Adjacent molecules interacting dispersively via aromatic rings exhibit total interaction energies of -22.6 to $-21.0 \mathrm{~kJ} \mathrm{~mol}^{-1}$, whereas other dispersive contacts are much higher in energy.

The energy computations also confirmed that dispersive interactions are by far the strongest influence. Electrostatic interactions are only of minor importance, whereas polarization does not play any role at all.

3.3.2. Hirshfeld surface and fingerprint plot. Amongst the many possibilities to partition and assign crystallographic space with respect to molecular crystals, a variation of Hirshfeld's stockholder partitioning (Hirshfeld, 1977) has become exceedingly popular. In this scheme, the surface assigned to a molecule encloses that volume in which the promolecule contribution from this molecule - constructed from nuclear positions and spherically averaged electron density - exceeds that of all neighbouring molecules (Spackman \& Byrom, 1997).

The information conveyed by shape representations of the Hirshfeld surface can be enhanced by colour-coding properties onto it. Fig. 4 (top) shows a projection of the normalized contact distance $d_{\text {norm }}$. Areas in which the distance between adjacent molecules is smaller and greater than the sum of their van der Waals radii are marked in red and blue, respectively. A first introspection already makes clear that most contacts are longer than (blue) or approximately equal to (white, especially along the molecular perimeter) the values expected from the van der Waals radii. Two exceptions are, however, distinguishable (red), viz. a side-on $\mathrm{C}-\mathrm{H} \cdots \pi$ contact between atom $\mathrm{H} 28 B$ and the $\mathrm{C} 21-\mathrm{C} 26$ ring (atom-plane distance = $2.544 \AA$ ) and a side-on $\mathrm{C}-\mathrm{H} \cdot \mathrm{H}-\mathrm{H}$ C contact between atoms $\mathrm{H} 23$ and $\mathrm{H} 13 A$ (distance $=2.314 \AA$ ).

The two-dimensional fingerprint plot of a Hirshfeld surface summarizes the intermolecular interactions in a crystal. For 

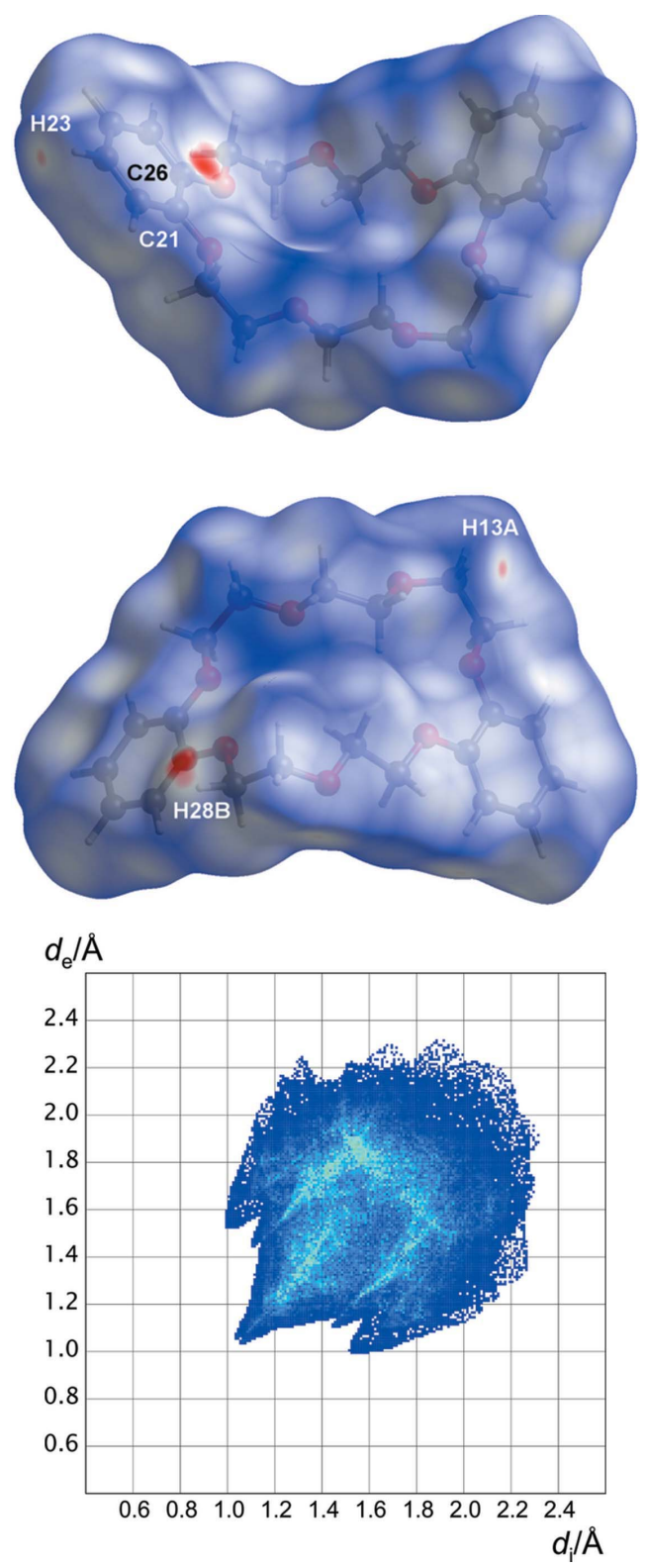

Figure 4

(Top) Hirshfeld surface with projection of the normalized contact distance $d_{\text {norm }}$ in two orientations (colour key: blue is positive, white is zero and red is negative). The molecule is shown in a ball-and-stick representation with arbitrary radii (colour key: $\mathrm{C}$ atoms black, $\mathrm{O}$ atoms red and $\mathrm{H}$ atoms white). (Bottom) A fingerprint plot of the Hirshfeld surface with external and internal distances, i.e. $d_{\mathrm{e}}$ and $d_{\mathrm{i}}$. The colours indicate the surface-area fraction, with a rainbow scheme ranging from blue (small) to red (large).

every point of the Hirshfeld surface, the distance $d_{\mathrm{i}}$ from the nearest nucleus inside versus the distance $d_{\mathrm{e}}$ from the nearest nucleus outside is plotted. Colour is used to encode the number of times each pair of distances occurs, equalling the respective surface-area fraction (see Fig. 4, bottom). The absence of colours in the range from yellow to red and the comparatively large spread $(1.0 \leq d \leq 2.3 \AA)$ show that the distance pairs are quite evenly distributed. This can be attributed to the anisotropic molecular shape giving rise to a variety of different contacts (Spackman \& McKinnon, 2002). Nevertheless, some features are discernible. The pale-green band roughly along the line defined by $d_{\mathrm{e}}=d_{\mathrm{i}}$ is due to the numerous dispersive head-to-head $\mathrm{H} \cdots \mathrm{H}$ interactions, while the pale-green bands below and above are caused by $\mathrm{C}-$ $\mathrm{H} \cdots \mathrm{O}$ interactions, i.e. the NCHBs. The blue wing-like structures, which are located even further out, result from $\mathrm{C}-$ $\mathrm{H} \cdots \pi$ contacts. The brightest feature at $d_{\mathrm{e}} \simeq 1.9 \AA, d_{\mathrm{i}} \simeq 1.5 \AA$ derives from an overlap of predominant $\mathrm{H} \cdots \mathrm{H}$ (between aliphatic, as well as between aliphatic and aromatic parts) and some $\mathrm{C}-\mathrm{H} \cdots \mathrm{O}$ interactions.

3.3.3. Electrostatic potential. Although electrostatic interactions are of minor importance ( $c f . \S 3.3 .1$ ), a short survey is in order for the sake of completeness. The electrostatic potential $V(\boldsymbol{r})$ at the point $\boldsymbol{r}$ is available from the final structural parameters and the predicted multipoles of an invariom refinement. For (I), it is illustrated in Fig. 5.

From the picture, it is evident that regions of high potential are located at the $\mathrm{H}$ atoms on the molecular perimeter, especially on the aromatic rings. Regions of low potential are located near the central fold dominated by $\mathrm{O}$ atoms, especially $\mathrm{O} 14$ and $\mathrm{O} 17$, and those stemming from catechol. It is safe to assume that what are overall relatively weak electrostatic interactions manifest primarily in NCHBs, as these are the predominant $\mathrm{C}-\mathrm{H} \cdots \mathrm{O}$ contacts. The findings are thus in tune with atom $\mathrm{O} 17$ accepting two hydrogen bonds, and O14 accepting one NCHB and having a relatively strong interaction with atom $\mathrm{H} 2 A$ ( $c f . \S 3.3 .1)$. The electrostatic interaction energies ( $c f$. Table S2 and Fig. S1 in the supporting information) nicely reflect the number of NCHBs between neighbouring molecules, as well as the adjacenct nature of regions of opposite potential: they are the lowest (unscaled $-29.7 \mathrm{~kJ} \mathrm{~mol}^{-1}$ ) for the stacks along $a$ and, remarkably second in place, for the dimers bound via hydrogen bonds of the shortest type (unscaled $-11.0 \mathrm{~kJ} \mathrm{~mol}^{-1}$ ).

\section{Conclusion}

Well-ordered solvent-free crystals of dibenzo-21-crown-7 can be grown from a solution in toluene via slow evaporation using a small temperature gradient. The tendency of crown ethers to form cocrystals with solvent molecules can be reduced using

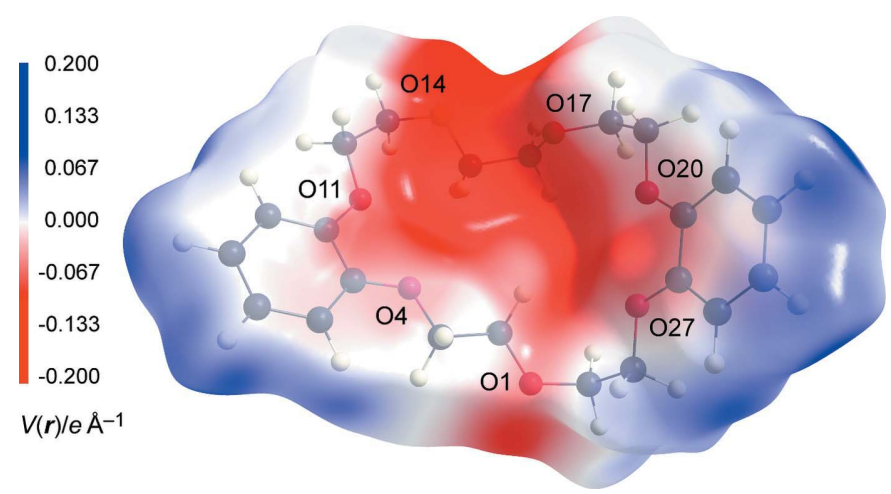

Figure 5

Electrostatic potential projected on the Hirshfeld surface. The molecule is shown in a ball-and-stick representation with arbitrary radii (colour key: $\mathrm{C}$ atoms black, $\mathrm{O}$ atoms red and $\mathrm{H}$ atoms white). 
apolar solvents. This is crucial in order not to disrupt dispersive contacts between crown-ether molecules in favour of stronger electrostatic interactions with solvent molecules.

The predominant intermolecular interactions in the crystal are of a dispersive nature and are manifested in various $\mathrm{C}-$ $\mathrm{H} \cdots \mathrm{H}-\mathrm{C}$ and $\mathrm{C}-\mathrm{H} \cdots \pi$ contacts. In addition, nonclassical $\mathrm{C}-\mathrm{H} \cdots \mathrm{O}$ hydrogen bonds are abundant, based on dispersive, as well as much weaker electrostatic interaction. The boat-like conformation of the molecules enables efficient stacking along the $a$ axis so that multiple weak dispersive contacts and hydrogen bonds contribute to a relatively strong total attraction between pairs of molecules in an infinite stack. Further, this motif seems to impose order even on a notoriously 'difficult group', such as the $-\mathrm{O}\left[\left(-\mathrm{CH}_{2}\right)_{2}-\mathrm{O}\right]_{3}-$ chain.

\section{Acknowledgements}

We thank Professor Andreas Grohmann (Technische Universität Berlin) for fruitful discussion, Dr Christian Hübschle (Universität Bayreuth) for help getting started with the MoleCoolQt and XD2016 software, and Ms Paula Nixdorf (Technische Universität Berlin) for collecting the diffraction data.

\section{References}

Blasius, E. \& Nilles, K.-H. (1984). Radiochim. Acta, 35, 173-182.

Burns, J. H., Bryan, J. C., Davis, M. C. \& Sachleben, R. A. (1996). J. Inclusion Phenom. Mol. Recognit. Chem. 26, 197-207.

Chemical Abstracts Service (2017). RN 14098-41-0. Columbus, Ohio, USA (accessed via SciFinder on July 7, 2017).

Desiraju, G. \& Steiner, T. (1999). In The Weak Hydrogen Bond in Structural Chemistry and Biology. Oxford University Press.

Dittrich, B., Hübschle, C. B., Pröpper, K., Dietrich, F., Stolper, T. \& Holstein, J. J. (2013). Acta Cryst. B69, 91-104.
Dittrich, B., Koritsánszky, T. \& Luger, P. (2004). Angew. Chem. Int. Ed. 43, 2718-2721.

Dolomanov, O. V., Bourhis, L. J., Gildea, R. J., Howard, J. A. K. \& Puschmann, H. (2009). J. Appl. Cryst. 42, 339-341.

Farrugia, L. J. (2012). J. Appl. Cryst. 45, 849-854.

Hansen, N. K. \& Coppens, P. (1978). Acta Cryst. A34, 909-921.

Hirshfeld, F. L. (1977). Theor. Chim. Acta, 44, 129-138.

Hübschle, C. B. \& Dittrich, B. (2011). J. Appl. Cryst. 44, 238-240.

Jayatilaka, D. \& Grimwood, D. J. (2003). Computational Science ICCS 2003, edited by P. M. A. Sloot, D. Abramson, A. V. Bogdanov, Y. E. Gorbachev, J. J. Dongarra \& A. Y. Zomaya, pp. 142-151. Berlin: Springer.

Mackenzie, C. F., Spackman, P. R., Jayatilaka, D. \& Spackman, M. A. (2017). IUCrJ, 4. https://doi.org/10.1107/S205225251700848X.

Macrae, C. F., Bruno, I. J., Chisholm, J. A., Edgington, P. R., McCabe, P., Pidcock, E., Rodriguez-Monge, L., Taylor, R., van de Streek, J. \& Wood, P. A. (2008). J. Appl. Cryst. 41, 466-470.

Mäkelä, T., Kiesilä, A., Kalenius, E. \& Rissanen, K. (2016). Chem. Eur. J. 22, 14264-14272.

Owen, J. D. \& Nowell, I. W. (1978). Acta Cryst. B34, 2354-2356.

Pedersen, C. J. (1967). J. Am. Chem. Soc. 89, 7017-7036.

Rigaku Oxford Diffraction (2015). CrysAlis PRO. Rigaku Corporation, Yarnton, Oxfordshire, England.

Sachleben, R. A., Bryan, J. C., Lavis, J. M., Starks, C. M. \& Burns, J. H. (1997). Tetrahedron, 53, 13567-13582.

Sanjuan-Szklarz, W. F., Hoser, A. A., Gutmann, M., Madsen, A. Ø. \& Wozniak, K. (2016). IUCrJ, 3, 61-70.

Sheldrick, G. M. (2015a). Acta Cryst. A71, 3-8.

Sheldrick, G. M. (2015b). Acta Cryst. C71, 3-8.

Spackman, M. A. \& Byrom, P. G. (1997). Chem. Phys. Lett. 267, $215-$ 220.

Spackman, M. A. \& McKinnon, J. J. (2002). CrystEngComm, 4, 378392.

Turner, M. J., McKinnon, J. J., Wolff, S. K., Grimwood, D. J., Spackman, P. R., Jayatilaka, D. \& Spackman, M. A. (2017). CrystalExplorer. University of Western Australia, Australia.

Volkov, A., Macchi, P., Farrugia, L. J., Gatti, C., Mallinson, P., Richter, T. \& Koritsánszky, T. (2016). XD2016. University of New York at Buffalo, New York, USA. 


\section{supporting information}

Acta Cryst. (2017). C73, 654-659 [https://doi.org/10.1107/S2053229617011160]

\section{Invariom-model refinement and Hirshfeld surface analysis of well-ordered solvent-free dibenzo-21-crown-7}

\section{Dennis Wiedemann and Julia Kohl}

\section{Computing details}

For both structures, data collection: CrysAlis PRO (Rigaku Oxford Diffraction, 2015); cell refinement: CrysAlis PRO (Rigaku Oxford Diffraction, 2015); data reduction: CrysAlis PRO (Rigaku Oxford Diffraction, 2015); program(s) used to solve structure: SHELXT (Sheldrick, 2015a). Program(s) used to refine structure: XD2016 (Volkov et al., 2016) for Invariom; SHELXL2016 (Sheldrick, 2015b) for IAM. For both structures, molecular graphics: ORTEP-3 for Windows (Farrugia, 2012) and Mercury (Macrae et al., 2008). Software used to prepare material for publication: WinXD (Volkov et al., 2016) for Invariom; OLEX2 (Dolomanov et al., 2009) for IAM.

Dibenzo[b,k][1,4,7,10,13,16,19]heptaoxacyclohenicosa-2,11-diene (Invariom)

\section{Crystal data}

\section{$\mathrm{C}_{22} \mathrm{H}_{28} \mathrm{O}_{7}$}

$M_{r}=404.44$

Monoclinic, $P 2{ }_{1} / c$

$a=4.9801(1) \AA$

$b=17.4771(2) \AA$

$c=23.1000(2) \AA$

$\beta=94.124(1)^{\circ}$

$V=2005.37(5) \AA^{3}$

$Z=4$

$F(000)=864$

\section{Data collection}

Agilent SuperNova (single source) diffractometer

Radiation source: micro-focus sealed tube, Agilent Nova

Mirror monochromator

Detector resolution: 10.5435 pixels $\mathrm{mm}^{-1}$

$\omega$ scans

Absorption correction: gaussian (CrysAlis PRO; Rigaku Oxford Diffraction, 2015)

Refinement

Refinement on $F^{2}$

Least-squares matrix: full

$R\left[F^{2}>2 \sigma\left(F^{2}\right)\right]=0.030$

$w R\left(F^{2}\right)=0.084$
$D_{\mathrm{x}}=1.34 \mathrm{Mg} \mathrm{m}^{-3}$

Melting point: $388 \mathrm{~K}$

$\mathrm{Cu} K \alpha$ radiation, $\lambda=1.54184 \AA$

Cell parameters from 7438 reflections

$\theta=2.5-73.5^{\circ}$

$\mu=0.82 \mathrm{~mm}^{-1}$

$T=150 \mathrm{~K}$

Shard, colourless

$0.74 \times 0.14 \times 0.08 \mathrm{~mm}$

$T_{\min }=0.598, T_{\max }=1.000$

13236 measured reflections

3925 independent reflections

3559 reflections with $I>2 \sigma(I)$

$R_{\text {int }}=0.018$

$\theta_{\max }=73.7^{\circ}, \theta_{\min }=3.2^{\circ}$

$h=-5 \rightarrow 6$

$k=-21 \rightarrow 21$

$l=-27 \rightarrow 28$

$S=0.98$

3664 reflections

290 parameters

0 restraints 
Primary atom site location: dual

Hydrogen site location: difference Fourier map

Only H-atom displacement parameters refined

$$
\begin{aligned}
& w=1 /\left[\sigma^{2}\left(F_{\mathrm{o}}^{2}\right)+(0.0497 P)^{2}+0.1 P\right] \\
& \quad \text { where } P=\left(F_{\mathrm{o}}^{2}+2 F_{\mathrm{c}}^{2}\right) / 3 \\
& (\Delta / \sigma)_{\max }<0.001 \\
& \Delta \rho_{\max }=0.41 \mathrm{e} \AA^{-3} \\
& \Delta \rho_{\min }=-0.24 \mathrm{e} \AA^{-3}
\end{aligned}
$$

Special details

Refinement. After conventional IAM refinement, invarioms were assigned and set up using MoleCoolQt Revision 558

\begin{tabular}{|c|c|c|c|c|}
\hline & $x$ & $y$ & $z$ & $U_{\text {iso }} * / U_{\text {eq }}$ \\
\hline $\mathrm{C} 2$ & $1.0446(2)$ & $0.65479(6)$ & $0.55315(5)$ & 0.027 \\
\hline $\mathrm{H} 2 \mathrm{~A}$ & 1.15515 & 0.70408 & 0.537643 & $0.056(5)^{*}$ \\
\hline $\mathrm{H} 2 \mathrm{~B}$ & 0.921845 & 0.673982 & 0.587914 & $0.051(4)^{*}$ \\
\hline $\mathrm{C} 3$ & $0.8678(2)$ & $0.61989(6)$ & $0.50382(5)$ & 0.026 \\
\hline $\mathrm{H} 3 \mathrm{~A}$ & 0.99184 & 0.597868 & 0.470203 & $0.040(4)^{*}$ \\
\hline H3B & 0.749237 & 0.572587 & 0.519914 & $0.042(4)^{*}$ \\
\hline $\mathrm{C} 5$ & $0.5121(2)$ & $0.66085(6)$ & $0.43710(4)$ & 0.024 \\
\hline C6 & $0.4908(2)$ & $0.58977(6)$ & $0.41032(5)$ & 0.027 \\
\hline H6 & 0.628561 & 0.544207 & 0.423852 & $0.043(4)^{*}$ \\
\hline $\mathrm{C} 7$ & $0.2899(2)$ & $0.57697(7)$ & $0.36562(5)$ & 0.03 \\
\hline $\mathrm{H} 7$ & 0.275295 & 0.52187 & 0.344238 & $0.053(4)^{*}$ \\
\hline $\mathrm{C} 8$ & $0.1099(2)$ & $0.63436(7)$ & $0.34872(5)$ & 0.031 \\
\hline $\mathrm{H} 8$ & -0.046729 & 0.623725 & 0.314726 & $0.057(5)^{*}$ \\
\hline C9 & $0.1296(2)$ & $0.70607(6)$ & $0.37537(5)$ & 0.028 \\
\hline H9 & -0.011815 & 0.750858 & 0.362114 & $0.051(4)^{*}$ \\
\hline $\mathrm{C} 10$ & $0.3311(2)$ & $0.71984(6)$ & $0.41890(4)$ & 0.024 \\
\hline $\mathrm{C} 12$ & $0.1553(2)$ & $0.84115(7)$ & $0.44408(5)$ & 0.033 \\
\hline $\mathrm{H} 12 \mathrm{~A}$ & -0.023216 & 0.814777 & 0.460837 & $0.061(5)^{*}$ \\
\hline H12B & 0.104154 & 0.859291 & 0.399212 & $0.060(5)^{*}$ \\
\hline $\mathrm{C} 13$ & 0.2414 & $0.90954(7)$ & $0.48096(5)$ & 0.037 \\
\hline $\mathrm{H} 13 \mathrm{~A}$ & 0.424768 & 0.933229 & 0.46439 & $0.064(5)^{*}$ \\
\hline H13B & 0.08429 & 0.953382 & 0.475337 & $0.064(5)^{*}$ \\
\hline $\mathrm{C} 15$ & $0.5459(3)$ & $0.85914(8)$ & $0.55510(6)$ & 0.041 \\
\hline $\mathrm{H} 15 \mathrm{~A}$ & 0.69461 & 0.880486 & 0.526282 & $0.075(6)^{*}$ \\
\hline H15B & 0.528137 & 0.797078 & 0.549115 & $0.081(6)^{*}$ \\
\hline $\mathrm{C} 16$ & $0.6417(3)$ & $0.87561(8)$ & $0.61654(6)$ & 0.04 \\
\hline H16A & 0.854031 & 0.859333 & 0.623821 & $0.092(7)^{*}$ \\
\hline H16B & 0.624433 & 0.937083 & 0.624937 & $0.075(6)^{*}$ \\
\hline $\mathrm{C} 18$ & $0.5696(3)$ & $0.84899(7)$ & $0.71319(6)$ & 0.039 \\
\hline H18A & 0.450759 & 0.896087 & 0.729498 & $0.069(6)^{*}$ \\
\hline H18B & 0.781893 & 0.866109 & 0.716492 & $0.066(5)^{*}$ \\
\hline C19 & $0.5354(2)$ & $0.77896(6)$ & $0.74960(5)$ & 0.032 \\
\hline H19A & 0.561502 & 0.793497 & 0.795824 & $0.054(4)^{*}$ \\
\hline H19B & 0.33372 & 0.754776 & 0.740426 & $0.057(5)^{*}$ \\
\hline
\end{tabular}
(Hübschle \& Dittrich, 2011).

All hydrogen atoms were reset to neutron-diffraction-derived distances to their carrier atoms after every refinement cycle $(\mathrm{d}=1.0962 \AA$ for methylene groups, $\mathrm{d}=1.0823 \AA$ for aromatics $)$.

Fractional atomic coordinates and isotropic or equivalent isotropic displacement parameters $\left(\AA^{2}\right)$ 


$\begin{array}{lllll}\mathrm{C} 21 & 0.7616(2) & 0.66022(6) & 0.76552(5) & 0.026 \\ \mathrm{C} 22 & 0.6192(2) & 0.64273(6) & 0.81330(5) & 0.03 \\ \mathrm{H} 22 & 0.476384 & 0.683409 & 0.828543 & 0.054(4)^{*} \\ \mathrm{C} 23 & 0.6593(2) & 0.57272(7) & 0.84169(5) & 0.034 \\ \mathrm{H} 23 & 0.547514 & 0.559374 & 0.87888 & 0.059(5)^{*} \\ \mathrm{C} 24 & 0.8413(2) & 0.52029(7) & 0.82286(5) & 0.034 \\ \mathrm{H} 24 & 0.871798 & 0.466227 & 0.845215 & 0.050(4)^{*} \\ \mathrm{C} 25 & 0.9859(2) & 0.53724(6) & 0.77468(5) & 0.03 \\ \mathrm{H} 25 & 1.129405 & 0.496312 & 0.760065 & 0.055(4)^{*} \\ \mathrm{C} 26 & 0.9465(2) & 0.60623(6) & 0.74558(5) & 0.026 \\ \mathrm{C} 28 & 1.2705(2) & 0.57778(6) & 0.67776(5) & 0.026 \\ \mathrm{H} 28 \mathrm{~A} & 1.182808 & 0.521846 & 0.666116 & 0.045(4)^{*} \\ \mathrm{H} 28 \mathrm{~B} & 1.432227 & 0.569403 & 0.711938 & 0.043(4)^{*} \\ \mathrm{C} 29 & 1.3829(2) & 0.61374(7) & 0.62490(5) & 0.029 \\ \mathrm{H} 29 \mathrm{~A} & 1.391005 & 0.675946 & 0.630839 & 0.051(4)^{*} \\ \mathrm{H} 29 \mathrm{~B} & 1.589078 & 0.59329 & 0.621268 & 0.054(5)^{*} \\ \mathrm{O} 1 & 1.22374(18) & 0.59660(5) & 0.57328(4) & 0.037 \\ \mathrm{O} 4 & 0.69566(15) & 0.67931(4) & 0.48120(3) & 0.028 \\ \mathrm{O} 11 & 0.37270(15) & 0.78813(4) & 0.44647(3) & 0.03 \\ \text { O14 } & 0.29216(18) & 0.89459(5) & 0.54075(4) & 0.04 \\ \text { O17 } & 0.48593(19) & 0.83406(5) & 0.65485(4) & 0.041 \\ \text { O20 } & 0.73637(16) & 0.72575(4) & 0.73461(4) & 0.033 \\ \text { O27 } & 1.06943(15) & 0.62756(5) & 0.69748(3) & 0.031 \\ & & & & \end{array}$

Atomic displacement parameters $\left(\AA^{2}\right)$

\begin{tabular}{|c|c|c|c|c|c|c|}
\hline & $U^{11}$ & $U^{22}$ & $U^{33}$ & $U^{12}$ & $U^{13}$ & $U^{23}$ \\
\hline $\mathrm{C} 2$ & $0.0249(5)$ & $0.0283(5)$ & $0.0278(5)$ & $0.0033(4)$ & $-0.0007(4)$ & -0.0021 \\
\hline $\mathrm{C} 3$ & $0.0234(5)$ & $0.0272(5)$ & 0.0270 & 0.0028 & -0.0005 (4) & -0.0013 \\
\hline $\mathrm{C} 5$ & $0.0203(5)$ & $0.0241(5)$ & $0.0262(5)$ & 0.0025 & 0.0008 (4) & $-0.0020(4)$ \\
\hline C6 & $0.0228(5)$ & $0.0246(5)$ & $0.0330(6)$ & $0.0032(4)$ & -0.0010 & -0.0055 \\
\hline $\mathrm{C} 7$ & $0.0264(6)$ & $0.0293(6)$ & $0.0342(6)$ & $0.0012(4)$ & $-0.0033(4)$ & -0.0068 \\
\hline $\mathrm{C} 8$ & $0.0272(6)$ & $0.0326(6)$ & $0.0313(6)$ & $0.0014(4)$ & $-0.0051(4)$ & $-0.0021(5)$ \\
\hline C9 & $0.0264(5)$ & 0.0289 (6) & $0.0294(5)$ & $0.0031(4)$ & $-0.0027(4)$ & 0.0018 \\
\hline $\mathrm{C} 10$ & $0.0237(5)$ & $0.0234(5)$ & $0.0259(5)$ & $0.0038(4)$ & $0.0013(4)$ & $0.0006(4)$ \\
\hline $\mathrm{C} 12$ & $0.0358(6)$ & $0.0281(6)$ & $0.0340(6)$ & $0.0106(5)$ & 0.0025 & $-0.0001(5)$ \\
\hline $\mathrm{C} 13$ & $0.0508(8)$ & $0.0278(6)$ & $0.0342(6)$ & $0.0113(5)$ & 0.0060 & $-0.0023(5)$ \\
\hline $\mathrm{C} 15$ & 0.0394 & $0.0482(8)$ & $0.0350(7)$ & $0.0064(6)$ & 0.0050 & $-0.0052(6)$ \\
\hline $\mathrm{C} 16$ & $0.0397(7)$ & $0.0396(7)$ & $0.0394(7)$ & $-0.0005(5)$ & $0.0035(5)$ & $-0.0083(5)$ \\
\hline $\mathrm{C} 18$ & $0.0516(8)$ & 0.0280 & $0.0361(6)$ & $0.0036(5)$ & $-0.0010(5)$ & $-0.0021(5)$ \\
\hline C19 & $0.0353(6)$ & $0.0281(6)$ & $0.0319(6)$ & $0.0056(5)$ & $0.0031(5)$ & -0.0034 \\
\hline $\mathrm{C} 21$ & $0.0246(5)$ & $0.0245(5)$ & $0.0282(5)$ & $-0.0006(4)$ & 0.0025 & -0.0054 \\
\hline $\mathrm{C} 22$ & $0.0311(6)$ & $0.0280(5)$ & $0.0315(6)$ & -0.0019 & $0.0058(4)$ & -0.0041 \\
\hline $\mathrm{C} 23$ & $0.0366(6)$ & $0.0314(6)$ & $0.0339(6)$ & $-0.0043(5)$ & $0.0063(5)$ & $-0.0006(5)$ \\
\hline $\mathrm{C} 24$ & $0.0345(6)$ & $0.0286(6)$ & $0.0384(6)$ & $-0.0022(5)$ & $0.0023(5)$ & $0.0029(5)$ \\
\hline $\mathrm{C} 25$ & $0.0272(6)$ & 0.0269 (6) & $0.0362(6)$ & $0.0006(4)$ & 0.0005 & $-0.0007(5)$ \\
\hline $\mathrm{C} 26$ & $0.0216(5)$ & $0.0255(5)$ & $0.0300(5)$ & $0.0006(4)$ & $0.0001(4)$ & $-0.0042(4)$ \\
\hline $\mathrm{C} 28$ & $0.0200(5)$ & $0.0287(5)$ & $0.0303(5)$ & $0.0027(4)$ & $-0.0011(4)$ & -0.0049 (4) \\
\hline
\end{tabular}




\begin{tabular}{lllllll}
$\mathrm{C} 29$ & $0.0244(5)$ & $0.0330(6)$ & $0.0293(5)$ & $0.0034(4)$ & $-0.0004(4)$ & $-0.0023(4)$ \\
O1 & $0.0432(5)$ & $0.0342(4)$ & $0.0319(4)$ & $0.0131(4)$ & $-0.0102(4)$ & $-0.0083(3)$ \\
O4 & $0.0251(4)$ & $0.0261(4)$ & $0.0311(4)$ & $0.0045(3)$ & $-0.0034(3)$ & $-0.0041(3)$ \\
O11 & $0.0291(4)$ & $0.0252(4)$ & $0.0343(4)$ & $0.0055(3)$ & $-0.0003(3)$ & $-0.0034(3)$ \\
O14 & $0.0411(5)$ & $0.0465(5)$ & $0.0322(4)$ & $0.0101(4)$ & $0.0081(4)$ & $-0.0028(4)$ \\
O17 & $0.0489(5)$ & $0.0393(5)$ & $0.0349(5)$ & $-0.0029(4)$ & $-0.0011(4)$ & $0.0011(4)$ \\
O20 & $0.0358(4)$ & $0.0283(4)$ & $0.0353(4)$ & $0.0053(3)$ & $0.0093(3)$ & $-0.0001(3)$ \\
O27 & $0.0284(4)$ & $0.0310(4)$ & $0.0332(4)$ & $0.0059(3)$ & $0.0055(3)$ & $-0.0004(3)$ \\
\hline
\end{tabular}

Geometric parameters $\left(\AA,{ }^{\circ}\right)$

\begin{tabular}{|c|c|c|c|}
\hline $\mathrm{C} 2-\mathrm{H} 2 \mathrm{~A}$ & 1.0962 & $\mathrm{C} 16-\mathrm{H} 16 \mathrm{~A}$ & 1.0962 \\
\hline $\mathrm{C} 2-\mathrm{H} 2 \mathrm{~B}$ & 1.0962 & $\mathrm{C} 16-\mathrm{H} 16 \mathrm{~B}$ & 1.0963 \\
\hline $\mathrm{C} 2-\mathrm{C} 3$ & $1.5171(15)$ & $\mathrm{C} 16-\mathrm{O} 17$ & $1.4181(16)$ \\
\hline $\mathrm{C} 2-\mathrm{O} 1$ & $1.4095(13)$ & $\mathrm{C} 18-\mathrm{H} 18 \mathrm{~A}$ & 1.0962 \\
\hline $\mathrm{C} 3-\mathrm{H} 3 \mathrm{~A}$ & 1.0963 & $\mathrm{C} 18-\mathrm{H} 18 \mathrm{~B}$ & 1.0962 \\
\hline C3-H3B & 1.0963 & $\mathrm{C} 18-\mathrm{C} 19$ & $1.5015(17)$ \\
\hline $\mathrm{C} 3-\mathrm{O} 4$ & $1.4221(12)$ & $\mathrm{C} 18-\mathrm{O} 17$ & $1.4060(15)$ \\
\hline $\mathrm{C} 5-\mathrm{C} 6$ & $1.3885(15)$ & C19-H19A & 1.0962 \\
\hline $\mathrm{C} 5-\mathrm{C} 10$ & $1.4135(14)$ & C19-H19B & 1.0962 \\
\hline $\mathrm{C} 5-\mathrm{O} 4$ & $1.3577(12)$ & $\mathrm{C} 19-\mathrm{O} 20$ & $1.4270(13)$ \\
\hline $\mathrm{C} 6-\mathrm{H} 6$ & 1.0822 & $\mathrm{C} 21-\mathrm{C} 22$ & $1.3882(15)$ \\
\hline $\mathrm{C} 6-\mathrm{C} 7$ & $1.4033(15)$ & $\mathrm{C} 21-\mathrm{C} 26$ & $1.4184(14)$ \\
\hline $\mathrm{C} 7-\mathrm{H} 7$ & 1.0822 & $\mathrm{C} 21-\mathrm{O} 20$ & $1.3507(13)$ \\
\hline $\mathrm{C} 7-\mathrm{C} 8$ & $1.3827(16)$ & $\mathrm{C} 22-\mathrm{H} 22$ & 1.0822 \\
\hline $\mathrm{C} 8-\mathrm{H} 8$ & 1.0823 & $\mathrm{C} 22-\mathrm{C} 23$ & $1.3957(17)$ \\
\hline $\mathrm{C} 8-\mathrm{C} 9$ & $1.3966(16)$ & $\mathrm{C} 23-\mathrm{H} 23$ & 1.0823 \\
\hline $\mathrm{C} 9-\mathrm{H} 9$ & 1.0822 & $\mathrm{C} 23-\mathrm{C} 24$ & $1.3810(17)$ \\
\hline $\mathrm{C} 9-\mathrm{C} 10$ & $1.3896(15)$ & $\mathrm{C} 24-\mathrm{H} 24$ & 1.0823 \\
\hline $\mathrm{C} 10-\mathrm{O} 11$ & $1.3618(13)$ & $\mathrm{C} 24-\mathrm{C} 25$ & $1.3999(17)$ \\
\hline $\mathrm{C} 12-\mathrm{H} 12 \mathrm{~A}$ & 1.0963 & $\mathrm{C} 25-\mathrm{H} 25$ & 1.0823 \\
\hline $\mathrm{C} 12-\mathrm{H} 12 \mathrm{~B}$ & 1.0962 & $\mathrm{C} 25-\mathrm{C} 26$ & $1.3875(16)$ \\
\hline $\mathrm{C} 12-\mathrm{C} 13$ & $1.5120(17)$ & $\mathrm{C} 26-\mathrm{O} 27$ & $1.3589(13)$ \\
\hline $\mathrm{C} 12-\mathrm{O} 11$ & $1.4232(13)$ & $\mathrm{C} 28-\mathrm{H} 28 \mathrm{~A}$ & 1.0963 \\
\hline C13-H13A & 1.0962 & $\mathrm{C} 28-\mathrm{H} 28 \mathrm{~B}$ & 1.0962 \\
\hline $\mathrm{C} 13-\mathrm{H} 13 \mathrm{~B}$ & 1.0962 & $\mathrm{C} 28-\mathrm{C} 29$ & $1.5157(16)$ \\
\hline $\mathrm{C} 13-\mathrm{O} 14$ & $1.4105(15)$ & $\mathrm{C} 28-\mathrm{O} 27$ & $1.4258(12)$ \\
\hline C15-H15A & 1.0963 & $\mathrm{C} 29-\mathrm{H} 29 \mathrm{~A}$ & 1.0963 \\
\hline C15-H15B & 1.0962 & $\mathrm{C} 29-\mathrm{H} 29 \mathrm{~B}$ & 1.0962 \\
\hline $\mathrm{C} 15-\mathrm{C} 16$ & $1.4924(18)$ & $\mathrm{C} 29-\mathrm{O} 1$ & $1.4155(13)$ \\
\hline $\mathrm{C} 15-\mathrm{O} 14$ & $1.4248(16)$ & & \\
\hline $\mathrm{H} 2 \mathrm{~A}-\mathrm{C} 2-\mathrm{H} 2 \mathrm{~B}$ & 108.58 & $\mathrm{H} 16 \mathrm{~A}-\mathrm{C} 16-\mathrm{O} 17$ & 109.65 \\
\hline $\mathrm{H} 2 \mathrm{~A}-\mathrm{C} 2-\mathrm{C} 3$ & 110.40 & $\mathrm{H} 16 \mathrm{~B}-\mathrm{C} 16-\mathrm{O} 17$ & 109.76 \\
\hline $\mathrm{H} 2 \mathrm{~A}-\mathrm{C} 2-\mathrm{O} 1$ & 110.73 & $\mathrm{H} 18 \mathrm{~A}-\mathrm{C} 18-\mathrm{H} 18 \mathrm{~B}$ & 108.23 \\
\hline $\mathrm{H} 2 \mathrm{~B}-\mathrm{C} 2-\mathrm{C} 3$ & 110.43 & $\mathrm{H} 18 \mathrm{~A}-\mathrm{C} 18-\mathrm{C} 19$ & 109.50 \\
\hline $\mathrm{H} 2 \mathrm{~B}-\mathrm{C} 2-\mathrm{O} 1$ & 110.62 & $\mathrm{H} 18 \mathrm{~A}-\mathrm{C} 18-\mathrm{O} 17$ & 109.79 \\
\hline $\mathrm{C} 3-\mathrm{C} 2-\mathrm{O} 1$ & $106.08(8)$ & $\mathrm{H} 18 \mathrm{~B}-\mathrm{C} 18-\mathrm{C} 19$ & 109.34 \\
\hline
\end{tabular}




\begin{tabular}{|c|c|c|c|}
\hline $\mathrm{C} 2-\mathrm{C} 3-\mathrm{H} 3 \mathrm{~A}$ & 110.31 & $\mathrm{H} 18 \mathrm{~B}-\mathrm{C} 18-\mathrm{O} 17$ & 109.58 \\
\hline $\mathrm{C} 2-\mathrm{C} 3-\mathrm{H} 3 \mathrm{~B}$ & 110.26 & $\mathrm{C} 19-\mathrm{C} 18-\mathrm{O} 17$ & $110.36(10)$ \\
\hline $\mathrm{C} 2-\mathrm{C} 3-\mathrm{O} 4$ & $106.52(8)$ & $\mathrm{C} 18-\mathrm{C} 19-\mathrm{H} 19 \mathrm{~A}$ & 110.27 \\
\hline $\mathrm{H} 3 \mathrm{~A}-\mathrm{C} 3-\mathrm{H} 3 \mathrm{~B}$ & 108.67 & $\mathrm{C} 18-\mathrm{C} 19-\mathrm{H} 19 \mathrm{~B}$ & 110.20 \\
\hline $\mathrm{H} 3 \mathrm{~A}-\mathrm{C} 3-\mathrm{O} 4$ & 110.58 & $\mathrm{C} 18-\mathrm{C} 19-\mathrm{O} 20$ & $106.59(9)$ \\
\hline $\mathrm{H} 3 \mathrm{~B}-\mathrm{C} 3-\mathrm{O} 4$ & 110.49 & $\mathrm{H} 19 \mathrm{~A}-\mathrm{C} 19-\mathrm{H} 19 \mathrm{~B}$ & 108.69 \\
\hline $\mathrm{C} 6-\mathrm{C} 5-\mathrm{C} 10$ & $119.56(10)$ & $\mathrm{H} 19 \mathrm{~A}-\mathrm{C} 19-\mathrm{O} 20$ & 110.56 \\
\hline $\mathrm{C} 6-\mathrm{C} 5-\mathrm{O} 4$ & $124.93(9)$ & $\mathrm{H} 19 \mathrm{~B}-\mathrm{C} 19-\mathrm{O} 20$ & 110.53 \\
\hline $\mathrm{C} 10-\mathrm{C} 5-\mathrm{O} 4$ & $115.50(9)$ & $\mathrm{C} 22-\mathrm{C} 21-\mathrm{C} 26$ & $119.50(10)$ \\
\hline $\mathrm{C} 5-\mathrm{C} 6-\mathrm{H} 6$ & 120.20 & $\mathrm{C} 22-\mathrm{C} 21-\mathrm{O} 20$ & $125.01(10)$ \\
\hline $\mathrm{C} 5-\mathrm{C} 6-\mathrm{C} 7$ & $119.87(10)$ & $\mathrm{C} 26-\mathrm{C} 21-\mathrm{O} 20$ & $115.49(9)$ \\
\hline $\mathrm{H} 6-\mathrm{C} 6-\mathrm{C} 7$ & 119.93 & $\mathrm{C} 21-\mathrm{C} 22-\mathrm{H} 22$ & 119.95 \\
\hline $\mathrm{C} 6-\mathrm{C} 7-\mathrm{H} 7$ & 119.90 & $\mathrm{C} 21-\mathrm{C} 22-\mathrm{C} 23$ & $120.17(10)$ \\
\hline $\mathrm{C} 6-\mathrm{C} 7-\mathrm{C} 8$ & $120.38(10)$ & $\mathrm{H} 22-\mathrm{C} 22-\mathrm{C} 23$ & 119.88 \\
\hline $\mathrm{H} 7-\mathrm{C} 7-\mathrm{C} 8$ & 119.72 & $\mathrm{C} 22-\mathrm{C} 23-\mathrm{H} 23$ & 119.83 \\
\hline $\mathrm{C} 7-\mathrm{C} 8-\mathrm{H} 8$ & 119.93 & $\mathrm{C} 22-\mathrm{C} 23-\mathrm{C} 24$ & $120.58(11)$ \\
\hline $\mathrm{C} 7-\mathrm{C} 8-\mathrm{C} 9$ & $120.20(10)$ & $\mathrm{H} 23-\mathrm{C} 23-\mathrm{C} 24$ & 119.59 \\
\hline $\mathrm{H} 8-\mathrm{C} 8-\mathrm{C} 9$ & 119.87 & $\mathrm{C} 23-\mathrm{C} 24-\mathrm{H} 24$ & 120.11 \\
\hline $\mathrm{C} 8-\mathrm{C} 9-\mathrm{H} 9$ & 120.05 & $\mathrm{C} 23-\mathrm{C} 24-\mathrm{C} 25$ & $119.78(11)$ \\
\hline $\mathrm{C} 8-\mathrm{C} 9-\mathrm{C} 10$ & $119.90(10)$ & $\mathrm{H} 24-\mathrm{C} 24-\mathrm{C} 25$ & 120.10 \\
\hline $\mathrm{H} 9-\mathrm{C} 9-\mathrm{C} 10$ & 120.05 & $\mathrm{C} 24-\mathrm{C} 25-\mathrm{H} 25$ & 119.81 \\
\hline $\mathrm{C} 5-\mathrm{C} 10-\mathrm{C} 9$ & $120.06(10)$ & $\mathrm{C} 24-\mathrm{C} 25-\mathrm{C} 26$ & $120.41(11)$ \\
\hline $\mathrm{C} 5-\mathrm{C} 10-\mathrm{O} 11$ & $115.32(9)$ & $\mathrm{H} 25-\mathrm{C} 25-\mathrm{C} 26$ & 119.78 \\
\hline $\mathrm{C} 9-\mathrm{C} 10-\mathrm{O} 11$ & $124.61(9)$ & $\mathrm{C} 21-\mathrm{C} 26-\mathrm{C} 25$ & $119.56(10)$ \\
\hline $\mathrm{H} 12 \mathrm{~A}-\mathrm{C} 12-\mathrm{H} 12 \mathrm{~B}$ & 108.44 & $\mathrm{C} 21-\mathrm{C} 26-\mathrm{O} 27$ & $115.11(9)$ \\
\hline $\mathrm{H} 12 \mathrm{~A}-\mathrm{C} 12-\mathrm{C} 13$ & 109.75 & $\mathrm{C} 25-\mathrm{C} 26-\mathrm{O} 27$ & $125.33(10)$ \\
\hline $\mathrm{H} 12 \mathrm{~A}-\mathrm{C} 12-\mathrm{O} 11$ & 110.28 & $\mathrm{H} 28 \mathrm{~A}-\mathrm{C} 28-\mathrm{H} 28 \mathrm{~B}$ & 108.47 \\
\hline $\mathrm{H} 12 \mathrm{~B}-\mathrm{C} 12-\mathrm{C} 13$ & 109.95 & $\mathrm{H} 28 \mathrm{~A}-\mathrm{C} 28-\mathrm{C} 29$ & 109.61 \\
\hline $\mathrm{H} 12 \mathrm{~B}-\mathrm{C} 12-\mathrm{O} 11$ & 110.44 & $\mathrm{H} 28 \mathrm{~A}-\mathrm{C} 28-\mathrm{O} 27$ & 110.23 \\
\hline $\mathrm{C} 13-\mathrm{C} 12-\mathrm{O} 11$ & $107.97(10)$ & $\mathrm{H} 28 \mathrm{~B}-\mathrm{C} 28-\mathrm{C} 29$ & 109.93 \\
\hline $\mathrm{C} 12-\mathrm{C} 13-\mathrm{H} 13 \mathrm{~A}$ & 108.15 & $\mathrm{H} 28 \mathrm{~B}-\mathrm{C} 28-\mathrm{O} 27$ & 110.36 \\
\hline $\mathrm{C} 12-\mathrm{C} 13-\mathrm{H} 13 \mathrm{~B}$ & 108.33 & $\mathrm{C} 29-\mathrm{C} 28-\mathrm{O} 27$ & $108.23(9)$ \\
\hline $\mathrm{C} 12-\mathrm{C} 13-\mathrm{O} 14$ & $115.39(10)$ & $\mathrm{C} 28-\mathrm{C} 29-\mathrm{H} 29 \mathrm{~A}$ & 108.84 \\
\hline $\mathrm{H} 13 \mathrm{~A}-\mathrm{C} 13-\mathrm{H} 13 \mathrm{~B}$ & 107.46 & $\mathrm{C} 28-\mathrm{C} 29-\mathrm{H} 29 \mathrm{~B}$ & 109.19 \\
\hline $\mathrm{H} 13 \mathrm{~A}-\mathrm{C} 13-\mathrm{O} 14$ & 108.47 & $\mathrm{C} 28-\mathrm{C} 29-\mathrm{O} 1$ & $111.98(9)$ \\
\hline $\mathrm{H} 13 \mathrm{~B}-\mathrm{C} 13-\mathrm{O} 14$ & 108.76 & $\mathrm{H} 29 \mathrm{~A}-\mathrm{C} 29-\mathrm{H} 29 \mathrm{~B}$ & 107.85 \\
\hline $\mathrm{H} 15 \mathrm{~A}-\mathrm{C} 15-\mathrm{H} 15 \mathrm{~B}$ & 108.18 & $\mathrm{H} 29 \mathrm{~A}-\mathrm{C} 29-\mathrm{O} 1$ & 109.19 \\
\hline $\mathrm{H} 15 \mathrm{~A}-\mathrm{C} 15-\mathrm{C} 16$ & 109.15 & $\mathrm{H} 29 \mathrm{~B}-\mathrm{C} 29-\mathrm{O} 1$ & 109.72 \\
\hline $\mathrm{H} 15 \mathrm{~A}-\mathrm{C} 15-\mathrm{O} 14$ & 109.71 & $\mathrm{C} 2-\mathrm{O} 1-\mathrm{C} 29$ & $115.34(8)$ \\
\hline $\mathrm{H} 15 \mathrm{~B}-\mathrm{C} 15-\mathrm{C} 16$ & 109.15 & $\mathrm{C} 3-\mathrm{O} 4-\mathrm{C} 5$ & $117.24(8)$ \\
\hline $\mathrm{H} 15 \mathrm{~B}-\mathrm{C} 15-\mathrm{O} 14$ & 109.80 & $\mathrm{C} 10-\mathrm{O} 11-\mathrm{C} 12$ & $117.56(8)$ \\
\hline $\mathrm{C} 16-\mathrm{C} 15-\mathrm{O} 14$ & $110.79(10)$ & $\mathrm{C} 13-\mathrm{O} 14-\mathrm{C} 15$ & $113.69(9)$ \\
\hline $\mathrm{C} 15-\mathrm{C} 16-\mathrm{H} 16 \mathrm{~A}$ & 109.56 & $\mathrm{C} 16-\mathrm{O} 17-\mathrm{C} 18$ & $111.43(10)$ \\
\hline $\mathrm{C} 15-\mathrm{C} 16-\mathrm{H} 16 \mathrm{~B}$ & 109.48 & $\mathrm{C} 19-\mathrm{O} 20-\mathrm{C} 21$ & $117.68(9)$ \\
\hline $\mathrm{C} 15-\mathrm{C} 16-\mathrm{O} 17$ & $110.05(11)$ & $\mathrm{C} 26-\mathrm{O} 27-\mathrm{C} 28$ & $117.51(9)$ \\
\hline $\mathrm{H} 16 \mathrm{~A}-\mathrm{C} 16-\mathrm{H} 16 \mathrm{~B}$ & 108.31 & & \\
\hline $\mathrm{H} 2 \mathrm{~A}-\mathrm{C} 2-\mathrm{C} 3-\mathrm{H} 3 \mathrm{~A}$ & 63.05 & $\mathrm{C} 16-\mathrm{C} 15-\mathrm{O} 14-\mathrm{C} 13$ & $154.91(14)$ \\
\hline
\end{tabular}




\begin{tabular}{|c|c|}
\hline $\mathrm{H} 2 \mathrm{~A}-\mathrm{C} 2-\mathrm{C} 3-\mathrm{H} 3 \mathrm{~B}$ & -176.92 \\
\hline $\mathrm{H} 2 \mathrm{~A}-\mathrm{C} 2-\mathrm{C} 3-\mathrm{O} 4$ & -57.00 \\
\hline $\mathrm{H} 2 \mathrm{~A}-\mathrm{C} 2-\mathrm{O} 1-\mathrm{C} 29$ & 68.75 \\
\hline $\mathrm{H} 2 \mathrm{~B}-\mathrm{C} 2-\mathrm{C} 3-\mathrm{H} 3 \mathrm{~A}$ & -176.87 \\
\hline $\mathrm{H} 2 \mathrm{~B}-\mathrm{C} 2-\mathrm{C} 3-\mathrm{H} 3 \mathrm{~B}$ & -56.83 \\
\hline $\mathrm{H} 2 \mathrm{~B}-\mathrm{C} 2-\mathrm{C} 3-\mathrm{O} 4$ & 63.08 \\
\hline $\mathrm{H} 2 \mathrm{~B}-\mathrm{C} 2-\mathrm{O} 1-\mathrm{C} 29$ & -51.67 \\
\hline $\mathrm{O} 1-\mathrm{C} 2-\mathrm{C} 3-\mathrm{H} 3 \mathrm{~A}$ & -56.97 \\
\hline $\mathrm{O} 1-\mathrm{C} 2-\mathrm{C} 3-\mathrm{H} 3 \mathrm{~B}$ & 63.07 \\
\hline $\mathrm{C} 3-\mathrm{C} 2-\mathrm{O} 1-\mathrm{C} 29$ & $-171.45(12)$ \\
\hline $\mathrm{O} 1-\mathrm{C} 2-\mathrm{C} 3-\mathrm{O} 4$ & $-177.02(11)$ \\
\hline $\mathrm{C} 2-\mathrm{C} 3-\mathrm{O} 4-\mathrm{C} 5$ & $-178.27(12)$ \\
\hline $\mathrm{H} 3 \mathrm{~A}-\mathrm{C} 3-\mathrm{O} 4-\mathrm{C} 5$ & 61.85 \\
\hline $\mathrm{H} 3 \mathrm{~B}-\mathrm{C} 3-\mathrm{O} 4-\mathrm{C} 5$ & -58.50 \\
\hline $\mathrm{C} 10-\mathrm{C} 5-\mathrm{C} 6-\mathrm{H} 6$ & $179.91(16)$ \\
\hline $\mathrm{C} 10-\mathrm{C} 5-\mathrm{C} 6-\mathrm{C} 7$ & $-0.19(10)$ \\
\hline $\mathrm{C} 6-\mathrm{C} 5-\mathrm{C} 10-\mathrm{C} 9$ & $1.44(10)$ \\
\hline $\mathrm{C} 6-\mathrm{C} 5-\mathrm{C} 10-\mathrm{O} 11$ & $-178.08(14)$ \\
\hline $\mathrm{C} 6-\mathrm{C} 5-\mathrm{O} 4-\mathrm{C} 3$ & $-4.01(10)$ \\
\hline $\mathrm{O} 4-\mathrm{C} 5-\mathrm{C} 6-\mathrm{H} 6$ & -0.88 \\
\hline $\mathrm{O} 4-\mathrm{C} 5-\mathrm{C} 6-\mathrm{C} 7$ & $179.03(16)$ \\
\hline $\mathrm{C} 10-\mathrm{C} 5-\mathrm{O} 4-\mathrm{C} 3$ & $175.23(13)$ \\
\hline $\mathrm{O} 4-\mathrm{C} 5-\mathrm{C} 10-\mathrm{C} 9$ & $-177.85(14)$ \\
\hline $\mathrm{O} 4-\mathrm{C} 5-\mathrm{C} 10-\mathrm{O} 11$ & $2.63(8)$ \\
\hline $\mathrm{C} 5-\mathrm{C} 6-\mathrm{C} 7-\mathrm{H} 7$ & 178.98 \\
\hline $\mathrm{C} 5-\mathrm{C} 6-\mathrm{C} 7-\mathrm{C} 8$ & $-1.08(10)$ \\
\hline $\mathrm{H} 6-\mathrm{C} 6-\mathrm{C} 7-\mathrm{H} 7$ & -1.12 \\
\hline $\mathrm{H} 6-\mathrm{C} 6-\mathrm{C} 7-\mathrm{C} 8$ & 178.82 \\
\hline $\mathrm{C} 6-\mathrm{C} 7-\mathrm{C} 8-\mathrm{H} 8$ & -178.94 \\
\hline $\mathrm{C} 6-\mathrm{C} 7-\mathrm{C} 8-\mathrm{C} 9$ & $1.11(11)$ \\
\hline $\mathrm{H} 7-\mathrm{C} 7-\mathrm{C} 8-\mathrm{H} 8$ & 1.00 \\
\hline $\mathrm{H} 7-\mathrm{C} 7-\mathrm{C} 8-\mathrm{C} 9$ & -178.95 \\
\hline $\mathrm{C} 7-\mathrm{C} 8-\mathrm{C} 9-\mathrm{H} 9$ & $-179.89(17)$ \\
\hline $\mathrm{C} 7-\mathrm{C} 8-\mathrm{C} 9-\mathrm{C} 10$ & $0.15(11)$ \\
\hline $\mathrm{H} 8-\mathrm{C} 8-\mathrm{C} 9-\mathrm{H} 9$ & $0.15(10)$ \\
\hline $\mathrm{H} 8-\mathrm{C} 8-\mathrm{C} 9-\mathrm{C} 10$ & $-179.80(17)$ \\
\hline $\mathrm{C} 8-\mathrm{C} 9-\mathrm{C} 10-\mathrm{C} 5$ & $-1.42(10)$ \\
\hline $\mathrm{C} 8-\mathrm{C} 9-\mathrm{C} 10-\mathrm{O} 11$ & $178.05(17)$ \\
\hline $\mathrm{H} 9-\mathrm{C} 9-\mathrm{C} 10-\mathrm{C} 5$ & 178.62 \\
\hline $\mathrm{H} 9-\mathrm{C} 9-\mathrm{C} 10-\mathrm{O} 11$ & -1.91 \\
\hline $\mathrm{C} 5-\mathrm{C} 10-\mathrm{O} 11-\mathrm{C} 12$ & $-160.57(13)$ \\
\hline $\mathrm{C} 9-\mathrm{C} 10-\mathrm{O} 11-\mathrm{C} 12$ & $19.94(10)$ \\
\hline $\mathrm{H} 12 \mathrm{~A}-\mathrm{C} 12-\mathrm{C} 13-\mathrm{H} 13 \mathrm{~A}$ & 177.62 \\
\hline $\mathrm{H} 12 \mathrm{~A}-\mathrm{C} 12-\mathrm{C} 13-\mathrm{H} 13 \mathrm{~B}$ & -66.19 \\
\hline $\mathrm{H} 12 \mathrm{~A}-\mathrm{C} 12-\mathrm{C} 13-\mathrm{O} 14$ & 55.97 \\
\hline $\mathrm{H} 12 \mathrm{~A}-\mathrm{C} 12-\mathrm{O} 11-\mathrm{C} 10$ & 56.09 \\
\hline $\mathrm{H} 12 \mathrm{~B}-\mathrm{C} 12-\mathrm{C} 13-\mathrm{H} 13 \mathrm{~A}$ & -63.17 \\
\hline $\mathrm{H} 12 \mathrm{~B}-\mathrm{C} 12-\mathrm{C} 13-\mathrm{H} 13 \mathrm{~B}$ & 53.01 \\
\hline
\end{tabular}

\begin{tabular}{|c|c|}
\hline $\mathrm{O} 14-\mathrm{C} 15-\mathrm{C} 16-\mathrm{H} 16 \mathrm{~A}$ & -167.24 \\
\hline $\mathrm{O} 14-\mathrm{C} 15-\mathrm{C} 16-\mathrm{H} 16 \mathrm{~B}$ & -48.60 \\
\hline $\mathrm{O} 14-\mathrm{C} 15-\mathrm{C} 16-\mathrm{O} 17$ & $72.12(12)$ \\
\hline $\mathrm{C} 15-\mathrm{C} 16-\mathrm{O} 17-\mathrm{C} 18$ & $-179.38(14)$ \\
\hline $\mathrm{H} 16 \mathrm{~A}-\mathrm{C} 16-\mathrm{O} 17-\mathrm{C} 18$ & $60.04(11)$ \\
\hline $\mathrm{H} 16 \mathrm{~B}-\mathrm{C} 16-\mathrm{O} 17-\mathrm{C} 18$ & -58.83 \\
\hline $\mathrm{H} 18 \mathrm{~A}-\mathrm{C} 18-\mathrm{C} 19-\mathrm{H} 19 \mathrm{~A}$ & -47.69 \\
\hline $\mathrm{H} 18 \mathrm{~A}-\mathrm{C} 18-\mathrm{C} 19-\mathrm{H} 19 \mathrm{~B}$ & 72.30 \\
\hline $\mathrm{H} 18 \mathrm{~A}-\mathrm{C} 18-\mathrm{C} 19-\mathrm{O} 20$ & -167.74 \\
\hline $\mathrm{H} 18 \mathrm{~A}-\mathrm{C} 18-\mathrm{O} 17-\mathrm{C} 16$ & 92.57 \\
\hline $\mathrm{H} 18 \mathrm{~B}-\mathrm{C} 18-\mathrm{C} 19-\mathrm{H} 19 \mathrm{~A}$ & 70.74 \\
\hline $\mathrm{H} 18 \mathrm{~B}-\mathrm{C} 18-\mathrm{C} 19-\mathrm{H} 19 \mathrm{~B}$ & -169.27 \\
\hline $\mathrm{H} 18 \mathrm{~B}-\mathrm{C} 18-\mathrm{C} 19-\mathrm{O} 20$ & -49.31 \\
\hline $\mathrm{H} 18 \mathrm{~B}-\mathrm{C} 18-\mathrm{O} 17-\mathrm{C} 16$ & -26.18 \\
\hline $\mathrm{C} 19-\mathrm{C} 18-\mathrm{O} 17-\mathrm{C} 16$ & $-146.64(14)$ \\
\hline $\mathrm{O} 17-\mathrm{C} 18-\mathrm{C} 19-\mathrm{H} 19 \mathrm{~A}$ & -168.66 \\
\hline $\mathrm{O} 17-\mathrm{C} 18-\mathrm{C} 19-\mathrm{H} 19 \mathrm{~B}$ & -48.67 \\
\hline $\mathrm{O} 17-\mathrm{C} 18-\mathrm{C} 19-\mathrm{O} 20$ & $71.29(11)$ \\
\hline $\mathrm{C} 18-\mathrm{C} 19-\mathrm{O} 20-\mathrm{C} 21$ & $174.35(13)$ \\
\hline $\mathrm{H} 19 \mathrm{~A}-\mathrm{C} 19-\mathrm{O} 20-\mathrm{C} 21$ & 54.49 \\
\hline $\mathrm{H} 19 \mathrm{~B}-\mathrm{C} 19-\mathrm{O} 20-\mathrm{C} 21$ & -65.90 \\
\hline $\mathrm{C} 26-\mathrm{C} 21-\mathrm{C} 22-\mathrm{H} 22$ & -179.44 \\
\hline $\mathrm{C} 26-\mathrm{C} 21-\mathrm{C} 22-\mathrm{C} 23$ & $0.42(10)$ \\
\hline $\mathrm{C} 22-\mathrm{C} 21-\mathrm{C} 26-\mathrm{C} 25$ & $-1.08(10)$ \\
\hline $\mathrm{C} 22-\mathrm{C} 21-\mathrm{C} 26-\mathrm{O} 27$ & $177.96(15)$ \\
\hline $\mathrm{C} 22-\mathrm{C} 21-\mathrm{O} 20-\mathrm{C} 19$ & $-4.50(10)$ \\
\hline $\mathrm{O} 20-\mathrm{C} 21-\mathrm{C} 22-\mathrm{H} 22$ & -0.55 \\
\hline $\mathrm{O} 20-\mathrm{C} 21-\mathrm{C} 22-\mathrm{C} 23$ & $179.31(17)$ \\
\hline $\mathrm{C} 26-\mathrm{C} 21-\mathrm{O} 20-\mathrm{C} 19$ & $174.42(14)$ \\
\hline $\mathrm{O} 20-\mathrm{C} 21-\mathrm{C} 26-\mathrm{C} 25$ & $179.93(15)$ \\
\hline $\mathrm{O} 20-\mathrm{C} 21-\mathrm{C} 26-\mathrm{O} 27$ & $-1.03(9)$ \\
\hline $\mathrm{C} 21-\mathrm{C} 22-\mathrm{C} 23-\mathrm{H} 23$ & $-179.90(18)$ \\
\hline $\mathrm{C} 21-\mathrm{C} 22-\mathrm{C} 23-\mathrm{C} 24$ & $0.29(11)$ \\
\hline $\mathrm{H} 22-\mathrm{C} 22-\mathrm{C} 23-\mathrm{H} 23$ & $-0.04(10)$ \\
\hline $\mathrm{H} 22-\mathrm{C} 22-\mathrm{C} 23-\mathrm{C} 24$ & $-179.85(18)$ \\
\hline $\mathrm{C} 22-\mathrm{C} 23-\mathrm{C} 24-\mathrm{H} 24$ & $179.75(18)$ \\
\hline $\mathrm{C} 22-\mathrm{C} 23-\mathrm{C} 24-\mathrm{C} 25$ & $-0.35(11)$ \\
\hline $\mathrm{H} 23-\mathrm{C} 23-\mathrm{C} 24-\mathrm{H} 24$ & $-0.05(10)$ \\
\hline $\mathrm{H} 23-\mathrm{C} 23-\mathrm{C} 24-\mathrm{C} 25$ & $179.84(18)$ \\
\hline $\mathrm{C} 23-\mathrm{C} 24-\mathrm{C} 25-\mathrm{H} 25$ & $179.62(18)$ \\
\hline $\mathrm{C} 23-\mathrm{C} 24-\mathrm{C} 25-\mathrm{C} 26$ & $-0.32(11)$ \\
\hline $\mathrm{H} 24-\mathrm{C} 24-\mathrm{C} 25-\mathrm{H} 25$ & $-0.48(10)$ \\
\hline $\mathrm{H} 24-\mathrm{C} 24-\mathrm{C} 25-\mathrm{C} 26$ & $179.58(18)$ \\
\hline $\mathrm{C} 24-\mathrm{C} 25-\mathrm{C} 26-\mathrm{C} 21$ & $1.03(10)$ \\
\hline $\mathrm{C} 24-\mathrm{C} 25-\mathrm{C} 26-\mathrm{O} 27$ & $-177.91(17)$ \\
\hline $\mathrm{H} 25-\mathrm{C} 25-\mathrm{C} 26-\mathrm{C} 21$ & -178.92 \\
\hline $\mathrm{H} 25-\mathrm{C} 25-\mathrm{C} 26-\mathrm{O} 27$ & 2.15 \\
\hline $\mathrm{C} 21-\mathrm{C} 26-\mathrm{O} 27-\mathrm{C} 28$ & $175.91(13)$ \\
\hline
\end{tabular}




$\begin{array}{llll}\mathrm{H} 12 \mathrm{~B}-\mathrm{C} 12-\mathrm{C} 13-\mathrm{O} 14 & 175.18 & \mathrm{C} 25-\mathrm{C} 26-\mathrm{O} 27-\mathrm{C} 28 & -5.11(10) \\ \mathrm{H} 12 \mathrm{~B}-\mathrm{C} 12-\mathrm{O} 11-\mathrm{C} 10 & -63.75 & \mathrm{H} 28 \mathrm{~A}-\mathrm{C} 28-\mathrm{C} 29-\mathrm{H} 29 \mathrm{~A} & 156.43 \\ \mathrm{C} 13-\mathrm{C} 12-\mathrm{O} 11-\mathrm{C} 10 & 176.00(13) & \mathrm{H} 28 \mathrm{~A}-\mathrm{C} 28-\mathrm{C} 29-\mathrm{H} 29 \mathrm{~B} & -86.08 \\ \mathrm{O} 11-\mathrm{C} 12-\mathrm{C} 13-\mathrm{H} 13 \mathrm{~A} & 57.38 & \mathrm{H} 28 \mathrm{~A}-\mathrm{C} 28-\mathrm{C} 29-\mathrm{O} 1 & 35.63 \\ \mathrm{O} 11-\mathrm{C} 12-\mathrm{C} 13-\mathrm{H} 13 \mathrm{~B} & 173.57 & \mathrm{H} 28 \mathrm{~A}-\mathrm{C} 28-\mathrm{O} 27-\mathrm{C} 26 & 61.37 \\ \mathrm{O} 11-\mathrm{C} 12-\mathrm{C} 13-\mathrm{O} 14 & -64.27(11) & \mathrm{H} 28 \mathrm{~B}-\mathrm{C} 28-\mathrm{C} 29-\mathrm{H} 29 \mathrm{~A} & -84.43 \\ \mathrm{C} 12-\mathrm{C} 13-\mathrm{O} 14-\mathrm{C} 15 & 77.86(12) & \mathrm{H} 28 \mathrm{~B}-\mathrm{C} 28-\mathrm{C} 29-\mathrm{H} 29 \mathrm{~B} & 33.06 \\ \mathrm{H} 13 \mathrm{~A}-\mathrm{C} 13-\mathrm{O} 14-\mathrm{C} 15 & -43.62 & \mathrm{H} 28 \mathrm{~B}-\mathrm{C} 28-\mathrm{C} 29-\mathrm{O} 1 & 154.77 \\ \mathrm{H} 13 \mathrm{~B}-\mathrm{C} 13-\mathrm{O} 14-\mathrm{C} 15 & -160.21 & \mathrm{H} 28 \mathrm{~B}-\mathrm{C} 28-\mathrm{O} 27-\mathrm{C} 26 & -58.42 \\ \mathrm{H} 15 \mathrm{~A}-\mathrm{C} 15-\mathrm{C} 16-\mathrm{H} 16 \mathrm{~A} & -46.32 & \mathrm{C} 29-\mathrm{C} 28-\mathrm{O} 27-\mathrm{C} 26 & -178.76(12) \\ \mathrm{H} 15 \mathrm{~A}-\mathrm{C} 15-\mathrm{C} 16-\mathrm{H} 16 \mathrm{~B} & 72.32 & \mathrm{O} 27-\mathrm{C} 28-\mathrm{C} 29-\mathrm{H} 29 \mathrm{~A} & 36.17 \\ \mathrm{H} 15 \mathrm{~A}-\mathrm{C} 15-\mathrm{C} 16-\mathrm{O} 17 & -166.96 & \mathrm{O} 27-\mathrm{C} 28-\mathrm{C} 29-\mathrm{O} 1 & 153.66 \\ \mathrm{H} 15 \mathrm{~A}-\mathrm{C} 15-\mathrm{O} 14-\mathrm{C} 13 & 34.32 & \mathrm{C} 28-\mathrm{C} 29-\mathrm{O} 1-\mathrm{C} 2 & -84.63(10) \\ \mathrm{H} 15 \mathrm{~B}-\mathrm{C} 15-\mathrm{C} 16-\mathrm{H} 16 \mathrm{~A} & 71.73 & \mathrm{H} 29 \mathrm{~A}-\mathrm{C} 29-\mathrm{O} 1-\mathrm{C} 2 & 99.84(11) \\ \mathrm{H} 15 \mathrm{~B}-\mathrm{C} 15-\mathrm{C} 16-\mathrm{H} 16 \mathrm{~B} & -169.63 & \mathrm{H} 29 \mathrm{~B}-\mathrm{C} 29-\mathrm{O} 1-\mathrm{C} 2 & -20.76 \\ \mathrm{H} 15 \mathrm{~B}-\mathrm{C} 15-\mathrm{C} 16-\mathrm{O} 17 & -48.91 & & -138.75 \\ \mathrm{H} 15 \mathrm{~B}-\mathrm{C} 15-\mathrm{O} 14-\mathrm{C} 13 & -84.44 & & \end{array}$

Hydrogen-bond geometry $\left(A,{ }^{\circ}\right)$

\begin{tabular}{lllll}
\hline$D-\mathrm{H} \cdots A$ & $D-\mathrm{H}$ & $\mathrm{H} \cdots A$ & $D \cdots A$ & $D-\mathrm{H} \cdots A$ \\
\hline $\mathrm{C} 2-\mathrm{H} 2 A \cdots \mathrm{O} 11^{\mathrm{i}}$ & 1.10 & 2.85 & $3.8410(14)$ & 151 \\
$\mathrm{C} 6-\mathrm{H} 6 \cdots \mathrm{O} 1^{\mathrm{ii}}$ & 1.08 & 2.57 & $3.5631(13)$ & 153 \\
$\mathrm{C} 7-\mathrm{H} 7 \cdots \mathrm{O} 27^{\mathrm{iii}}$ & 1.08 & 3.23 & $4.2104(14)$ & 151 \\
$\mathrm{C} 13-\mathrm{H} 13 B \cdots \mathrm{O} 14^{\mathrm{iv}}$ & 1.10 & 3.26 & $4.3405(15)$ & 170 \\
$\mathrm{C} 16-\mathrm{H} 16 A \cdots \mathrm{O} 17^{\mathrm{i}}$ & 1.10 & 3.21 & $4.2956(16)$ & 172 \\
$\mathrm{C} 19-\mathrm{H} 19 B \cdots \mathrm{O} 20^{\mathrm{v}}$ & 1.10 & 3.01 & $4.0769(14)$ & 164 \\
$\mathrm{C} 29-\mathrm{H} 29 A \cdots \mathrm{O} 17^{\mathrm{i}}$ & 1.10 & 2.85 & $3.9393(15)$ & 172 \\
$\mathrm{C} 29-\mathrm{H} 29 B \cdots \mathrm{O} 1^{\mathrm{i}}$ & 1.10 & 3.42 & $4.4459(14)$ & 156 \\
\hline
\end{tabular}

Symmetry codes: (i) $x+1, y, z$; (ii) $-x+2,-y+1,-z+1$; (iii) $-x+1,-y+1,-z+1$; (iv) $-x,-y+2,-z+1$; (v) $x-1, y, z$.

Dibenzo[b,k][1,4,7,10,13,16,19]heptaoxacyclohenicosa-2,11-diene (IAM)

Crystal data

$\mathrm{C}_{22} \mathrm{H}_{28} \mathrm{O}_{7}$

$M_{r}=404.44$

Monoclinic, $P 2_{1} / c$

$a=4.9801(1) \AA$

$b=17.4771(2) \AA$

$c=23.1000(2) \AA$

$\beta=94.124(1)^{\circ}$

$V=2005.37(5) \AA^{3}$

$Z=4$

$F(000)=864$

Data collection

Agilent SuperNova (Single Source) diffractometer

Radiation source: micro-focus sealed tube, Agilent Nova
$D_{\mathrm{x}}=1.340 \mathrm{Mg} \mathrm{m}^{-3}$

Melting point: $388 \mathrm{~K}$

$\mathrm{Cu} K \alpha$ radiation, $\lambda=1.54184 \AA$

Cell parameters from 7438 reflections

$\theta=2.5-73.5^{\circ}$

$\mu=0.82 \mathrm{~mm}^{-1}$

$T=150 \mathrm{~K}$

Shard, colourless

$0.74 \times 0.14 \times 0.08 \mathrm{~mm}$

Mirror monochromator

Detector resolution: 10.5435 pixels $\mathrm{mm}^{-1}$ $\omega$ scans 
Absorption correction: gaussian

(CrysAlis PRO; Rigaku Oxford Diffraction, 2015)

$T_{\min }=0.598, T_{\max }=1.000$

13236 measured reflections

3925 independent reflections

Refinement

Refinement on $F^{2}$

Least-squares matrix: full

$R\left[F^{2}>2 \sigma\left(F^{2}\right)\right]=0.038$

$w R\left(F^{2}\right)=0.103$

$S=1.05$

3925 reflections

262 parameters

0 restraints
3559 reflections with $I>2 \sigma(I)$

$R_{\text {int }}=0.018$

$\theta_{\max }=73.7^{\circ}, \theta_{\min }=3.2^{\circ}$

$h=-5 \rightarrow 6$

$k=-21 \rightarrow 21$

$l=-27 \rightarrow 28$

Primary atom site location: dual

Hydrogen site location: difference Fourier map

$\mathrm{H}$-atom parameters constrained

$w=1 /\left[\sigma^{2}\left(F_{0}^{2}\right)+(0.0497 P)^{2}+0.7337 P\right]$

where $P=\left(F_{\mathrm{o}}^{2}+2 F_{\mathrm{c}}^{2}\right) / 3$

$(\Delta / \sigma)_{\max }<0.001$

$\Delta \rho_{\max }=0.39 \mathrm{e} \AA^{-3}$

$\Delta \rho_{\min }=-0.28$ e $\AA^{-3}$

Special details

Geometry. All esds (except the esd in the dihedral angle between two 1.s. planes) are estimated using the full covariance matrix. The cell esds are taken into account individually in the estimation of esds in distances, angles and torsion angles; correlations between esds in cell parameters are only used when they are defined by crystal symmetry. An approximate (isotropic) treatment of cell esds is used for estimating esds involving 1.s. planes.

Refinement. All hydrogen atoms were refined with standard riding models $(\mathrm{d}=0.99 \AA$ for methylene groups, $\mathrm{d}=0.95 \AA$ for aromatics) and $\mathrm{U}_{\mathrm{iso}}(\mathrm{H})=1.2 \times \mathrm{U}_{\mathrm{eq}}(\mathrm{C})$.

Fractional atomic coordinates and isotropic or equivalent isotropic displacement parameters $\left(\hat{A}^{2}\right)$

\begin{tabular}{lllll}
\hline & $x$ & $y$ & $z$ & $U_{\text {iso }} / U_{\text {eq }}$ \\
\hline C2 & $1.0441(3)$ & $0.65491(8)$ & $0.55310(6)$ & $0.0297(3)$ \\
H2A & 1.144473 & 0.699349 & 0.539126 & $0.036^{*}$ \\
H2B & 0.933591 & 0.672147 & 0.584572 & $0.036^{*}$ \\
C3 & $0.8681(3)$ & $0.61985(7)$ & $0.50392(6)$ & $0.0284(3)$ \\
H3A & 0.979783 & 0.600009 & 0.473489 & $0.034^{*}$ \\
H3B & 0.760709 & 0.577146 & 0.518369 & $0.034^{*}$ \\
C5 & $0.5116(2)$ & $0.66076(7)$ & $0.43693(5)$ & $0.0263(3)$ \\
C6 & $0.4900(3)$ & $0.58992(8)$ & $0.41022(6)$ & $0.0296(3)$ \\
H6 & 0.611055 & 0.549979 & 0.422126 & $0.036^{*}$ \\
C7 & $0.2897(3)$ & $0.57723(8)$ & $0.36571(6)$ & $0.0330(3)$ \\
H7 & 0.277119 & 0.528863 & 0.346947 & $0.040^{*}$ \\
C8 & $0.1107(3)$ & $0.63442(8)$ & $0.34888(6)$ & $0.0333(3)$ \\
H8 & -0.026797 & 0.625078 & 0.319046 & $0.040^{*}$ \\
C9 & $0.1303(3)$ & $0.70585(8)$ & $0.37543(6)$ & $0.0312(3)$ \\
H9 & 0.006182 & 0.745160 & 0.363804 & $0.037^{*}$ \\
C10 & $0.3313(3)$ & $0.71958(7)$ & $0.41884(5)$ & $0.0272(3)$ \\
C12 & $0.1548(3)$ & $0.84138(8)$ & $0.44420(6)$ & $0.0354(3)$ \\
H12A & -0.006134 & 0.817332 & 0.459237 & $0.042^{*}$ \\
H12B & 0.109109 & 0.857535 & 0.403608 & $0.042^{*}$ \\
C13 & $0.2412(3)$ & $0.90949(8)$ & $0.48085(6)$ & $0.0400(3)$ \\
H13A & 0.406919 & 0.930919 & 0.465993 & $0.048^{*}$ \\
H13B & 0.099447 & 0.949141 & 0.475868 & $0.048^{*}$ \\
C15 & $0.5467(3)$ & $0.85913(10)$ & $0.55533(7)$ & $0.0431(4)$ \\
& & & &
\end{tabular}




$\begin{array}{lllll}\text { H15A } & 0.680158 & 0.878400 & 0.529116 & 0.052^{*} \\ \text { H15B } & 0.529938 & 0.803124 & 0.549722 & 0.052^{*} \\ \text { C16 } & 0.6416(3) & 0.87554(9) & 0.61641(7) & 0.0418(4) \\ \text { H16A } & 0.833382 & 0.860914 & 0.623103 & 0.050^{*} \\ \text { H16B } & 0.626119 & 0.931038 & 0.624099 & 0.050^{*} \\ \text { C18 } & 0.5696(3) & 0.84884(9) & 0.71328(7) & 0.0413(3) \\ \text { H18A } & 0.462399 & 0.891458 & 0.727912 & 0.050^{*} \\ \text { H18B } & 0.761253 & 0.864425 & 0.716177 & 0.050^{*} \\ \text { C19 } & 0.5352(3) & 0.77915(8) & 0.74954(6) & 0.0343(3) \\ \text { H19A } & 0.558938 & 0.792103 & 0.791319 & 0.041^{*} \\ \text { H19B } & 0.353259 & 0.757138 & 0.741314 & 0.041^{*} \\ \text { C21 } & 0.7615(3) & 0.66002(7) & 0.76567(6) & 0.0284(3) \\ \text { C22 } & 0.6198(3) & 0.64255(8) & 0.81321(6) & 0.0326(3) \\ \text { H22 } & 0.494445 & 0.678260 & 0.826614 & 0.039^{*} \\ \text { C23 } & 0.6602(3) & 0.57274(8) & 0.84152(6) & 0.0362(3) \\ \text { H23 } & 0.561808 & 0.561065 & 0.874138 & 0.043^{*} \\ \text { C24 } & 0.8411(3) & 0.52054(8) & 0.82273(6) & 0.0363(3) \\ \text { H24 } & 0.867921 & 0.473103 & 0.842373 & 0.044^{*} \\ \text { C25 } & 0.9854(3) & 0.53739(8) & 0.77473(6) & 0.0329(3) \\ \text { H25 } & 1.111404 & 0.501449 & 0.761901 & 0.039^{*} \\ \text { C26 } & 0.9459(2) & 0.60618(7) & 0.74572(6) & 0.0283(3) \\ \text { C28 } & 1.2710(2) & 0.57773(8) & 0.67757(6) & 0.0290(3) \\ \text { H28A } & 1.191303 & 0.527226 & 0.667225 & 0.035^{*} \\ \text { H28B } & 1.416418 & 0.570221 & 0.708571 & 0.035^{*} \\ \text { C29 } & 1.3829(3) & 0.61383(8) & 0.62507(6) & 0.0316(3) \\ \text { H29A } & 1.390228 & 0.670029 & 0.630294 & 0.038^{*} \\ \text { H29B } & 1.568907 & 0.595295 & 0.621641 & 0.038^{*} \\ \text { O1 } & 1.2240(2) & 0.59634(6) & 0.57316(4) & 0.0395(3) \\ \text { O4 } & 0.69569(18) & 0.67957(5) & 0.48126(4) & 0.0302(2) \\ \text { O11 } & 0.37362(19) & 0.78814(5) & 0.44656(4) & 0.0323(2) \\ \text { O14 } & 0.2914(2) & 0.89471(6) & 0.54092(4) & 0.0422(3) \\ \text { O17 } & 0.4851(2) & 0.83391(6) & 0.65474(5) & 0.0439(3) \\ \text { O20 } & 0.7371(2) & 0.72584(5) & 0.73445(4) & 0.0354(2) \\ \text { O27 } & 1.06917(19) & 0.62778(5) & 0.69739(4) & 0.0331(2) \\ & & & & \end{array}$

Atomic displacement parameters $\left(\AA^{2}\right)$

\begin{tabular}{lllllll}
\hline & $U^{11}$ & $U^{22}$ & $U^{33}$ & $U^{12}$ & $U^{13}$ & $U^{23}$ \\
\hline C2 & $0.0276(6)$ & $0.0300(7)$ & $0.0313(7)$ & $0.0043(5)$ & $0.0006(5)$ & $-0.0009(5)$ \\
C3 & $0.0262(6)$ & $0.0282(6)$ & $0.0304(6)$ & $0.0042(5)$ & $0.0004(5)$ & $-0.0001(5)$ \\
C5 & $0.0219(6)$ & $0.0304(6)$ & $0.0266(6)$ & $0.0000(5)$ & $0.0025(5)$ & $-0.0008(5)$ \\
C6 & $0.0249(6)$ & $0.0283(6)$ & $0.0357(7)$ & $0.0041(5)$ & $0.0018(5)$ & $-0.0027(5)$ \\
C7 & $0.0302(7)$ & $0.0320(7)$ & $0.0365(7)$ & $-0.0008(5)$ & $0.0008(5)$ & $-0.0068(6)$ \\
C8 & $0.0286(7)$ & $0.0388(8)$ & $0.0317(7)$ & $-0.0012(5)$ & $-0.0030(5)$ & $-0.0013(6)$ \\
C9 & $0.0282(7)$ & $0.0329(7)$ & $0.0321(7)$ & $0.0039(5)$ & $0.0001(5)$ & $0.0042(5)$ \\
C10 & $0.0281(6)$ & $0.0262(6)$ & $0.0278(6)$ & $0.0012(5)$ & $0.0054(5)$ & $-0.0002(5)$ \\
C12 & $0.0371(7)$ & $0.0312(7)$ & $0.0378(7)$ & $0.0121(6)$ & $0.0031(6)$ & $0.0015(6)$ \\
C13 & $0.0529(9)$ & $0.0323(7)$ & $0.0353(7)$ & $0.0120(7)$ & $0.0066(6)$ & $-0.0001(6)$
\end{tabular}




\begin{tabular}{lllllll} 
C15 & $0.0406(8)$ & $0.0492(9)$ & $0.0399(8)$ & $0.0075(7)$ & $0.0060(6)$ & $-0.0043(7)$ \\
C16 & $0.0408(8)$ & $0.0411(8)$ & $0.0440(8)$ & $-0.0008(6)$ & $0.0056(6)$ & $-0.0073(6)$ \\
C18 & $0.0532(9)$ & $0.0323(7)$ & $0.0377(8)$ & $0.0043(7)$ & $-0.0015(7)$ & $-0.0036(6)$ \\
C19 & $0.0366(7)$ & $0.0316(7)$ & $0.0349(7)$ & $0.0064(6)$ & $0.0038(6)$ & $-0.0052(6)$ \\
C21 & $0.0273(6)$ & $0.0262(6)$ & $0.0313(6)$ & $-0.0026(5)$ & $-0.0014(5)$ & $-0.0049(5)$ \\
C22 & $0.0318(7)$ & $0.0317(7)$ & $0.0345(7)$ & $-0.0029(5)$ & $0.0041(5)$ & $-0.0071(5)$ \\
C23 & $0.0376(8)$ & $0.0368(8)$ & $0.0344(7)$ & $-0.0071(6)$ & $0.0046(6)$ & $-0.0021(6)$ \\
C24 & $0.0367(7)$ & $0.0310(7)$ & $0.0406(8)$ & $-0.0044(6)$ & $-0.0015(6)$ & $0.0026(6)$ \\
C25 & $0.0278(7)$ & $0.0304(7)$ & $0.0400(7)$ & $0.0003(5)$ & $-0.0013(5)$ & $-0.0034(6)$ \\
C26 & $0.0236(6)$ & $0.0303(7)$ & $0.0305(6)$ & $-0.0028(5)$ & $-0.0014(5)$ & $-0.0048(5)$ \\
C28 & $0.0218(6)$ & $0.0309(7)$ & $0.0338(7)$ & $0.0037(5)$ & $-0.0015(5)$ & $-0.0061(5)$ \\
C29 & $0.0270(7)$ & $0.0353(7)$ & $0.0320(7)$ & $0.0037(5)$ & $-0.0017(5)$ & $-0.0038(5)$ \\
O1 & $0.0458(6)$ & $0.0367(5)$ & $0.0338(5)$ & $0.0139(4)$ & $-0.0116(4)$ & $-0.0084(4)$ \\
O4 & $0.0272(5)$ & $0.0287(5)$ & $0.0338(5)$ & $0.0050(4)$ & $-0.0042(4)$ & $-0.0042(4)$ \\
O11 & $0.0319(5)$ & $0.0267(5)$ & $0.0378(5)$ & $0.0061(4)$ & $-0.0005(4)$ & $-0.0043(4)$ \\
O14 & $0.0436(6)$ & $0.0504(6)$ & $0.0335(5)$ & $0.0113(5)$ & $0.0078(4)$ & $-0.0021(5)$ \\
O17 & $0.0525(6)$ & $0.0431(6)$ & $0.0355(5)$ & $-0.0040(5)$ & $-0.0007(5)$ & $0.0012(4)$ \\
O20 & $0.0387(5)$ & $0.0302(5)$ & $0.0383(5)$ & $0.0064(4)$ & $0.0099(4)$ & $0.0006(4)$ \\
O27 & $0.0310(5)$ & $0.0335(5)$ & $0.0353(5)$ & $0.0063(4)$ & $0.0068(4)$ & $-0.0003(4)$ \\
& & & & & & \\
\hline
\end{tabular}

Geometric parameters $\left(A,{ }^{\circ}\right)$

\begin{tabular}{llll}
\hline $\mathrm{C} 2-\mathrm{H} 2 \mathrm{~A}$ & 0.9900 & $\mathrm{C} 16-\mathrm{H} 16 \mathrm{~A}$ & 0.9900 \\
$\mathrm{C} 2-\mathrm{H} 2 \mathrm{~B}$ & 0.9900 & $\mathrm{C} 16-\mathrm{H} 16 \mathrm{~B}$ & 0.9900 \\
$\mathrm{C} 2-\mathrm{C} 3$ & $1.5138(18)$ & $\mathrm{C} 16-\mathrm{O} 17$ & $1.4214(19)$ \\
$\mathrm{C} 2-\mathrm{O} 1$ & $1.4160(16)$ & $\mathrm{C} 18-\mathrm{H} 18 \mathrm{~A}$ & 0.9900 \\
$\mathrm{C} 3-\mathrm{H} 3 \mathrm{~A}$ & 0.9900 & $\mathrm{C} 18-\mathrm{H} 18 \mathrm{~B}$ & 0.9900 \\
$\mathrm{C} 3-\mathrm{H} 3 \mathrm{~B}$ & 0.9900 & $\mathrm{C} 18-\mathrm{C} 19$ & $1.495(2)$ \\
$\mathrm{C} 3-\mathrm{O} 4$ & $1.4267(15)$ & $\mathrm{C} 18-\mathrm{O} 17$ & $1.4114(18)$ \\
$\mathrm{C} 5-\mathrm{C} 6$ & $1.3841(18)$ & $\mathrm{C} 19-\mathrm{H} 19 \mathrm{~A}$ & 0.9900 \\
$\mathrm{C} 5-\mathrm{C} 10$ & $1.4085(18)$ & $\mathrm{C} 19-\mathrm{H} 19 \mathrm{~B}$ & 0.9900 \\
$\mathrm{C} 5-\mathrm{O} 4$ & $1.3646(15)$ & $\mathrm{C} 19-\mathrm{O} 20$ & $1.4321(16)$ \\
$\mathrm{C} 6-\mathrm{H} 6$ & 0.9500 & $\mathrm{C} 21-\mathrm{C} 22$ & $1.3817(19)$ \\
$\mathrm{C} 6-\mathrm{C} 7$ & $1.3977(19)$ & $\mathrm{C} 21-\mathrm{C} 26$ & $1.4151(18)$ \\
$\mathrm{C} 7-\mathrm{H} 7$ & 0.9500 & $\mathrm{C} 21-\mathrm{O} 20$ & $1.3585(16)$ \\
$\mathrm{C} 7-\mathrm{C} 8$ & $1.377(2)$ & $\mathrm{C} 22-\mathrm{H} 22$ & 0.9500 \\
$\mathrm{C} 8-\mathrm{H} 8$ & 0.9500 & $\mathrm{C} 22-\mathrm{C} 23$ & $1.392(2)$ \\
$\mathrm{C} 8-\mathrm{C} 9$ & $1.391(2)$ & $\mathrm{C} 23-\mathrm{H} 23$ & 0.9500 \\
$\mathrm{C} 9-\mathrm{H} 9$ & 0.9500 & $\mathrm{C} 23-\mathrm{C} 24$ & $1.374(2)$ \\
$\mathrm{C} 9-\mathrm{C} 10$ & $1.3858(19)$ & $\mathrm{C} 24-\mathrm{H} 24$ & 0.9500 \\
$\mathrm{C} 10-\mathrm{O} 11$ & $1.3678(16)$ & $\mathrm{C} 24-\mathrm{C} 25$ & $1.395(2)$ \\
$\mathrm{C} 12-\mathrm{H} 12 \mathrm{~A}$ & $\mathrm{C} 25-\mathrm{H} 25$ & 0.9500 \\
$\mathrm{C} 12-\mathrm{H} 12 \mathrm{~B}$ & 0.9900 & $\mathrm{C} 25-\mathrm{C} 26$ & $1.3834(19)$ \\
$\mathrm{C} 12-\mathrm{C} 13$ & 0.9900 & $\mathrm{C} 26-\mathrm{O} 27$ & $1.3654(16)$ \\
$\mathrm{C} 12-\mathrm{O} 11$ & $1.505(2)$ & $\mathrm{C} 28-\mathrm{H} 28 \mathrm{~A}$ & 0.9900 \\
$\mathrm{C} 13-\mathrm{H} 13 \mathrm{~A}$ & $1.4308(15)$ & $\mathrm{C} 28-\mathrm{H} 28 \mathrm{~B}$ & 0.9900 \\
$\mathrm{C} 13-\mathrm{H} 13 \mathrm{~B}$ & 0.9900 & $\mathrm{C} 28-\mathrm{C} 29$ & $1.5087(19)$ \\
$\mathrm{C} 13-\mathrm{O} 14$ & 0.9900 & & $1.4321(15)$ \\
& $1.4159(18)$ & &
\end{tabular}




\begin{tabular}{|c|c|c|c|}
\hline $\mathrm{C} 15-\mathrm{H} 15 \mathrm{~A}$ & 0.9900 & $\mathrm{C} 29-\mathrm{H} 29 \mathrm{~A}$ & 0.9900 \\
\hline $\mathrm{C} 15-\mathrm{H} 15 \mathrm{~B}$ & 0.9900 & $\mathrm{C} 29-\mathrm{H} 29 \mathrm{~B}$ & 0.9900 \\
\hline $\mathrm{C} 15-\mathrm{C} 16$ & $1.483(2)$ & $\mathrm{C} 29-\mathrm{O} 1$ & $1.4216(16)$ \\
\hline $\mathrm{C} 15-\mathrm{O} 14$ & $1.4328(19)$ & & \\
\hline $\mathrm{H} 2 \mathrm{~A}-\mathrm{C} 2-\mathrm{H} 2 \mathrm{~B}$ & 108.7 & $\mathrm{O} 17-\mathrm{C} 16-\mathrm{H} 16 \mathrm{~A}$ & 109.7 \\
\hline $\mathrm{C} 3-\mathrm{C} 2-\mathrm{H} 2 \mathrm{~A}$ & 110.6 & $\mathrm{O} 17-\mathrm{C} 16-\mathrm{H} 16 \mathrm{~B}$ & 109.7 \\
\hline $\mathrm{C} 3-\mathrm{C} 2-\mathrm{H} 2 \mathrm{~B}$ & 110.6 & $\mathrm{H} 18 \mathrm{~A}-\mathrm{C} 18-\mathrm{H} 18 \mathrm{~B}$ & 108.1 \\
\hline $\mathrm{O} 1-\mathrm{C} 2-\mathrm{H} 2 \mathrm{~A}$ & 110.6 & $\mathrm{C} 19-\mathrm{C} 18-\mathrm{H} 18 \mathrm{~A}$ & 109.6 \\
\hline $\mathrm{O} 1-\mathrm{C} 2-\mathrm{H} 2 \mathrm{~B}$ & 110.6 & $\mathrm{C} 19-\mathrm{C} 18-\mathrm{H} 18 \mathrm{~B}$ & 109.6 \\
\hline $\mathrm{O} 1-\mathrm{C} 2-\mathrm{C} 3$ & $105.76(10)$ & $\mathrm{O} 17-\mathrm{C} 18-\mathrm{H} 18 \mathrm{~A}$ & 109.6 \\
\hline $\mathrm{C} 2-\mathrm{C} 3-\mathrm{H} 3 \mathrm{~A}$ & 110.5 & $\mathrm{O} 17-\mathrm{C} 18-\mathrm{H} 18 \mathrm{~B}$ & 109.6 \\
\hline $\mathrm{C} 2-\mathrm{C} 3-\mathrm{H} 3 \mathrm{~B}$ & 110.5 & $\mathrm{O} 17-\mathrm{C} 18-\mathrm{C} 19$ & $110.35(12)$ \\
\hline $\mathrm{H} 3 \mathrm{~A}-\mathrm{C} 3-\mathrm{H} 3 \mathrm{~B}$ & 108.7 & $\mathrm{C} 18-\mathrm{C} 19-\mathrm{H} 19 \mathrm{~A}$ & 110.4 \\
\hline $\mathrm{O} 4-\mathrm{C} 3-\mathrm{C} 2$ & $106.26(10)$ & $\mathrm{C} 18-\mathrm{C} 19-\mathrm{H} 19 \mathrm{~B}$ & 110.4 \\
\hline $\mathrm{O} 4-\mathrm{C} 3-\mathrm{H} 3 \mathrm{~A}$ & 110.5 & $\mathrm{H} 19 \mathrm{~A}-\mathrm{C} 19-\mathrm{H} 19 \mathrm{~B}$ & 108.6 \\
\hline $\mathrm{O} 4-\mathrm{C} 3-\mathrm{H} 3 \mathrm{~B}$ & 110.5 & $\mathrm{O} 20-\mathrm{C} 19-\mathrm{C} 18$ & $106.42(12)$ \\
\hline $\mathrm{C} 6-\mathrm{C} 5-\mathrm{C} 10$ & $119.54(12)$ & $\mathrm{O} 20-\mathrm{C} 19-\mathrm{H} 19 \mathrm{~A}$ & 110.4 \\
\hline $\mathrm{O} 4-\mathrm{C} 5-\mathrm{C} 6$ & $125.21(12)$ & $\mathrm{O} 20-\mathrm{C} 19-\mathrm{H} 19 \mathrm{~B}$ & 110.4 \\
\hline $\mathrm{O} 4-\mathrm{C} 5-\mathrm{C} 10$ & $115.24(11)$ & $\mathrm{C} 22-\mathrm{C} 21-\mathrm{C} 26$ & $119.51(12)$ \\
\hline $\mathrm{C} 5-\mathrm{C} 6-\mathrm{H} 6$ & 120.1 & $\mathrm{O} 20-\mathrm{C} 21-\mathrm{C} 22$ & $125.29(12)$ \\
\hline $\mathrm{C} 5-\mathrm{C} 6-\mathrm{C} 7$ & $119.89(12)$ & $\mathrm{O} 20-\mathrm{C} 21-\mathrm{C} 26$ & $115.20(12)$ \\
\hline $\mathrm{C} 7-\mathrm{C} 6-\mathrm{H} 6$ & 120.1 & $\mathrm{C} 21-\mathrm{C} 22-\mathrm{H} 22$ & 119.9 \\
\hline $\mathrm{C} 6-\mathrm{C} 7-\mathrm{H} 7$ & 119.8 & $\mathrm{C} 21-\mathrm{C} 22-\mathrm{C} 23$ & $120.14(13)$ \\
\hline $\mathrm{C} 8-\mathrm{C} 7-\mathrm{C} 6$ & $120.39(13)$ & $\mathrm{C} 23-\mathrm{C} 22-\mathrm{H} 22$ & 119.9 \\
\hline $\mathrm{C} 8-\mathrm{C} 7-\mathrm{H} 7$ & 119.8 & $\mathrm{C} 22-\mathrm{C} 23-\mathrm{H} 23$ & 119.7 \\
\hline $\mathrm{C} 7-\mathrm{C} 8-\mathrm{H} 8$ & 119.9 & $\mathrm{C} 24-\mathrm{C} 23-\mathrm{C} 22$ & $120.62(13)$ \\
\hline $\mathrm{C} 7-\mathrm{C} 8-\mathrm{C} 9$ & $120.23(12)$ & $\mathrm{C} 24-\mathrm{C} 23-\mathrm{H} 23$ & 119.7 \\
\hline $\mathrm{C} 9-\mathrm{C} 8-\mathrm{H} 8$ & 119.9 & $\mathrm{C} 23-\mathrm{C} 24-\mathrm{H} 24$ & 120.1 \\
\hline $\mathrm{C} 8-\mathrm{C} 9-\mathrm{H} 9$ & 120.0 & $\mathrm{C} 23-\mathrm{C} 24-\mathrm{C} 25$ & $119.86(13)$ \\
\hline $\mathrm{C} 10-\mathrm{C} 9-\mathrm{C} 8$ & $119.91(12)$ & $\mathrm{C} 25-\mathrm{C} 24-\mathrm{H} 24$ & 120.1 \\
\hline $\mathrm{C} 10-\mathrm{C} 9-\mathrm{H} 9$ & 120.0 & $\mathrm{C} 24-\mathrm{C} 25-\mathrm{H} 25$ & 119.8 \\
\hline $\mathrm{C} 9-\mathrm{C} 10-\mathrm{C} 5$ & $120.01(12)$ & $\mathrm{C} 26-\mathrm{C} 25-\mathrm{C} 24$ & $120.31(13)$ \\
\hline $\mathrm{O} 11-\mathrm{C} 10-\mathrm{C} 5$ & $115.27(11)$ & $\mathrm{C} 26-\mathrm{C} 25-\mathrm{H} 25$ & 119.8 \\
\hline $\mathrm{O} 11-\mathrm{C} 10-\mathrm{C} 9$ & $124.71(12)$ & $\mathrm{C} 25-\mathrm{C} 26-\mathrm{C} 21$ & $119.55(13)$ \\
\hline $\mathrm{H} 12 \mathrm{~A}-\mathrm{C} 12-\mathrm{H} 12 \mathrm{~B}$ & 108.4 & $\mathrm{O} 27-\mathrm{C} 26-\mathrm{C} 21$ & $115.01(12)$ \\
\hline $\mathrm{C} 13-\mathrm{C} 12-\mathrm{H} 12 \mathrm{~A}$ & 110.1 & $\mathrm{O} 27-\mathrm{C} 26-\mathrm{C} 25$ & $125.43(12)$ \\
\hline $\mathrm{C} 13-\mathrm{C} 12-\mathrm{H} 12 \mathrm{~B}$ & 110.1 & $\mathrm{H} 28 \mathrm{~A}-\mathrm{C} 28-\mathrm{H} 28 \mathrm{~B}$ & 108.4 \\
\hline $\mathrm{O} 11-\mathrm{C} 12-\mathrm{H} 12 \mathrm{~A}$ & 110.1 & $\mathrm{C} 29-\mathrm{C} 28-\mathrm{H} 28 \mathrm{~A}$ & 110.1 \\
\hline $\mathrm{O} 11-\mathrm{C} 12-\mathrm{H} 12 \mathrm{~B}$ & 110.1 & $\mathrm{C} 29-\mathrm{C} 28-\mathrm{H} 28 \mathrm{~B}$ & 110.1 \\
\hline $\mathrm{O} 11-\mathrm{C} 12-\mathrm{C} 13$ & $107.87(12)$ & $\mathrm{O} 27-\mathrm{C} 28-\mathrm{H} 28 \mathrm{~A}$ & 110.1 \\
\hline $\mathrm{C} 12-\mathrm{C} 13-\mathrm{H} 13 \mathrm{~A}$ & 108.4 & $\mathrm{O} 27-\mathrm{C} 28-\mathrm{H} 28 \mathrm{~B}$ & 110.1 \\
\hline $\mathrm{C} 12-\mathrm{C} 13-\mathrm{H} 13 \mathrm{~B}$ & 108.4 & $\mathrm{O} 27-\mathrm{C} 28-\mathrm{C} 29$ & $108.05(11)$ \\
\hline $\mathrm{H} 13 \mathrm{~A}-\mathrm{C} 13-\mathrm{H} 13 \mathrm{~B}$ & 107.5 & $\mathrm{C} 28-\mathrm{C} 29-\mathrm{H} 29 \mathrm{~A}$ & 109.2 \\
\hline $\mathrm{O} 14-\mathrm{C} 13-\mathrm{C} 12$ & $115.47(12)$ & $\mathrm{C} 28-\mathrm{C} 29-\mathrm{H} 29 \mathrm{~B}$ & 109.2 \\
\hline $\mathrm{O} 14-\mathrm{C} 13-\mathrm{H} 13 \mathrm{~A}$ & 108.4 & $\mathrm{H} 29 \mathrm{~A}-\mathrm{C} 29-\mathrm{H} 29 \mathrm{~B}$ & 107.9 \\
\hline $\mathrm{O} 14-\mathrm{C} 13-\mathrm{H} 13 \mathrm{~B}$ & 108.4 & $\mathrm{O} 1-\mathrm{C} 29-\mathrm{C} 28$ & $111.89(11)$ \\
\hline $\mathrm{H} 15 \mathrm{~A}-\mathrm{C} 15-\mathrm{H} 15 \mathrm{~B}$ & 108.1 & $\mathrm{O} 1-\mathrm{C} 29-\mathrm{H} 29 \mathrm{~A}$ & 109.2 \\
\hline
\end{tabular}




\begin{tabular}{|c|c|c|c|}
\hline $\mathrm{C} 16-\mathrm{C} 15-\mathrm{H} 15 \mathrm{~A}$ & 109.5 & $\mathrm{O} 1-\mathrm{C} 29-\mathrm{H} 29 \mathrm{~B}$ & 109.2 \\
\hline $\mathrm{C} 16-\mathrm{C} 15-\mathrm{H} 15 \mathrm{~B}$ & 109.5 & $\mathrm{C} 2-\mathrm{O} 1-\mathrm{C} 29$ & $114.92(10)$ \\
\hline $\mathrm{O} 14-\mathrm{C} 15-\mathrm{H} 15 \mathrm{~A}$ & 109.5 & $\mathrm{C} 5-\mathrm{O} 4-\mathrm{C} 3$ & $116.94(10)$ \\
\hline $\mathrm{O} 14-\mathrm{C} 15-\mathrm{H} 15 \mathrm{~B}$ & 109.5 & $\mathrm{C} 10-\mathrm{O} 11-\mathrm{C} 12$ & $117.42(10)$ \\
\hline $\mathrm{O} 14-\mathrm{C} 15-\mathrm{C} 16$ & $110.73(12)$ & $\mathrm{C} 13-\mathrm{O} 14-\mathrm{C} 15$ & $113.46(11)$ \\
\hline $\mathrm{C} 15-\mathrm{C} 16-\mathrm{H} 16 \mathrm{~A}$ & 109.7 & $\mathrm{C} 18-\mathrm{O} 17-\mathrm{C} 16$ & $111.29(12)$ \\
\hline $\mathrm{C} 15-\mathrm{C} 16-\mathrm{H} 16 \mathrm{~B}$ & 109.7 & $\mathrm{C} 21-\mathrm{O} 20-\mathrm{C} 19$ & $117.34(11)$ \\
\hline $\mathrm{H} 16 \mathrm{~A}-\mathrm{C} 16-\mathrm{H} 16 \mathrm{~B}$ & 108.2 & $\mathrm{C} 26-\mathrm{O} 27-\mathrm{C} 28$ & $117.35(10)$ \\
\hline $\mathrm{O} 17-\mathrm{C} 16-\mathrm{C} 15$ & $109.99(13)$ & & \\
\hline $\mathrm{C} 2-\mathrm{C} 3-\mathrm{O} 4-\mathrm{C} 5$ & $-178.30(10)$ & $\mathrm{C} 22-\mathrm{C} 21-\mathrm{C} 26-\mathrm{O} 27$ & $177.94(11)$ \\
\hline $\mathrm{C} 3-\mathrm{C} 2-\mathrm{O} 1-\mathrm{C} 29$ & $-171.31(11)$ & $\mathrm{C} 22-\mathrm{C} 21-\mathrm{O} 20-\mathrm{C} 19$ & $-4.54(19)$ \\
\hline $\mathrm{C} 5-\mathrm{C} 6-\mathrm{C} 7-\mathrm{C} 8$ & $-1.1(2)$ & $\mathrm{C} 22-\mathrm{C} 23-\mathrm{C} 24-\mathrm{C} 25$ & $-0.2(2)$ \\
\hline $\mathrm{C} 5-\mathrm{C} 10-\mathrm{O} 11-\mathrm{C} 12$ & $-160.54(11)$ & $\mathrm{C} 23-\mathrm{C} 24-\mathrm{C} 25-\mathrm{C} 26$ & $-0.4(2)$ \\
\hline $\mathrm{C} 6-\mathrm{C} 5-\mathrm{C} 10-\mathrm{C} 9$ & $1.36(19)$ & $\mathrm{C} 24-\mathrm{C} 25-\mathrm{C} 26-\mathrm{C} 21$ & $1.10(19)$ \\
\hline $\mathrm{C} 6-\mathrm{C} 5-\mathrm{C} 10-\mathrm{O} 11$ & $-178.08(11)$ & $\mathrm{C} 24-\mathrm{C} 25-\mathrm{C} 26-\mathrm{O} 27$ & $-177.90(12)$ \\
\hline $\mathrm{C} 6-\mathrm{C} 5-\mathrm{O} 4-\mathrm{C} 3$ & $-3.98(18)$ & $\mathrm{C} 25-\mathrm{C} 26-\mathrm{O} 27-\mathrm{C} 28$ & $-5.00(18)$ \\
\hline $\mathrm{C} 6-\mathrm{C} 7-\mathrm{C} 8-\mathrm{C} 9$ & $1.1(2)$ & $\mathrm{C} 26-\mathrm{C} 21-\mathrm{C} 22-\mathrm{C} 23$ & $0.55(19)$ \\
\hline $\mathrm{C} 7-\mathrm{C} 8-\mathrm{C} 9-\mathrm{C} 10$ & $0.2(2)$ & $\mathrm{C} 26-\mathrm{C} 21-\mathrm{O} 20-\mathrm{C} 19$ & $174.39(11)$ \\
\hline $\mathrm{C} 8-\mathrm{C} 9-\mathrm{C} 10-\mathrm{C} 5$ & $-1.4(2)$ & $\mathrm{C} 28-\mathrm{C} 29-\mathrm{O} 1-\mathrm{C} 2$ & $100.04(14)$ \\
\hline $\mathrm{C} 8-\mathrm{C} 9-\mathrm{C} 10-\mathrm{O} 11$ & $177.99(12)$ & $\mathrm{C} 29-\mathrm{C} 28-\mathrm{O} 27-\mathrm{C} 26$ & $-178.62(10)$ \\
\hline $\mathrm{C} 9-\mathrm{C} 10-\mathrm{O} 11-\mathrm{C} 12$ & $20.06(18)$ & $\mathrm{O} 1-\mathrm{C} 2-\mathrm{C} 3-\mathrm{O} 4$ & $-176.91(10)$ \\
\hline $\mathrm{C} 10-\mathrm{C} 5-\mathrm{C} 6-\mathrm{C} 7$ & $-0.11(19)$ & $\mathrm{O} 4-\mathrm{C} 5-\mathrm{C} 6-\mathrm{C} 7$ & $179.02(12)$ \\
\hline $\mathrm{C} 10-\mathrm{C} 5-\mathrm{O} 4-\mathrm{C} 3$ & $175.18(11)$ & $\mathrm{O} 4-\mathrm{C} 5-\mathrm{C} 10-\mathrm{C} 9$ & $-177.86(11)$ \\
\hline $\mathrm{C} 12-\mathrm{C} 13-\mathrm{O} 14-\mathrm{C} 15$ & $78.08(17)$ & $\mathrm{O} 4-\mathrm{C} 5-\mathrm{C} 10-\mathrm{O} 11$ & $2.71(16)$ \\
\hline $\mathrm{C} 13-\mathrm{C} 12-\mathrm{O} 11-\mathrm{C} 10$ & $176.07(11)$ & $\mathrm{O} 11-\mathrm{C} 12-\mathrm{C} 13-\mathrm{O} 14$ & $-64.62(16)$ \\
\hline $\mathrm{C} 15-\mathrm{C} 16-\mathrm{O} 17-\mathrm{C} 18$ & $-179.56(12)$ & $\mathrm{O} 14-\mathrm{C} 15-\mathrm{C} 16-\mathrm{O} 17$ & $72.03(17)$ \\
\hline $\mathrm{C} 16-\mathrm{C} 15-\mathrm{O} 14-\mathrm{C} 13$ & $155.01(14)$ & $\mathrm{O} 17-\mathrm{C} 18-\mathrm{C} 19-\mathrm{O} 20$ & $71.48(15)$ \\
\hline $\mathrm{C} 18-\mathrm{C} 19-\mathrm{O} 20-\mathrm{C} 21$ & $174.35(11)$ & $\mathrm{O} 20-\mathrm{C} 21-\mathrm{C} 22-\mathrm{C} 23$ & $179.43(12)$ \\
\hline $\mathrm{C} 19-\mathrm{C} 18-\mathrm{O} 17-\mathrm{C} 16$ & $-146.68(13)$ & $\mathrm{O} 20-\mathrm{C} 21-\mathrm{C} 26-\mathrm{C} 25$ & $179.85(11)$ \\
\hline $\mathrm{C} 21-\mathrm{C} 22-\mathrm{C} 23-\mathrm{C} 24$ & $0.1(2)$ & $\mathrm{O} 20-\mathrm{C} 21-\mathrm{C} 26-\mathrm{O} 27$ & $-1.05(16)$ \\
\hline $\mathrm{C} 21-\mathrm{C} 26-\mathrm{O} 27-\mathrm{C} 28$ & $175.96(11)$ & $\mathrm{O} 27-\mathrm{C} 28-\mathrm{C} 29-\mathrm{O} 1$ & $-85.10(13)$ \\
\hline $\mathrm{C} 22-\mathrm{C} 21-\mathrm{C} 26-\mathrm{C} 25$ & $-1.16(19)$ & & \\
\hline
\end{tabular}

Hydrogen-bond geometry $\left(\AA,{ }^{o}\right)$

\begin{tabular}{lllll}
\hline$D-\mathrm{H} \cdots A$ & $D-\mathrm{H}$ & $\mathrm{H} \cdots A$ & $D \cdots A$ & $D-\mathrm{H} \cdots A$ \\
\hline $\mathrm{C} 2-\mathrm{H} 2 A \cdots \mathrm{O} 11^{\mathrm{i}}$ & 0.99 & 2.94 & $3.8408(17)$ & 152 \\
$\mathrm{C} 6-\mathrm{H} 6 \cdots \mathrm{O} 1^{\mathrm{ii}}$ & 0.95 & 2.69 & $3.5630(16)$ & 154 \\
$\mathrm{C} 7-\mathrm{H} 7 \cdots \mathrm{O} 27^{\mathrm{iii}}$ & 0.95 & 3.36 & $4.2170(17)$ & 152 \\
$\mathrm{C} 13-\mathrm{H} 13 B \cdots \mathrm{O} 14^{\mathrm{iv}}$ & 0.99 & 3.36 & $4.3366(18)$ & 170 \\
$\mathrm{C} 16-\mathrm{H} 16 A \cdots \mathrm{O} 17^{\mathrm{i}}$ & 0.99 & 3.31 & $4.292(2)$ & 172 \\
$\mathrm{C} 19-\mathrm{H} 19 B \cdots \mathrm{O} 20^{v}$ & 0.99 & 3.11 & $4.0729(18)$ & 165 \\
$\mathrm{C} 29-\mathrm{H} 29 A \cdots \mathrm{O} 17^{\mathrm{i}}$ & 0.99 & 2.95 & $3.9337(18)$ & 172 \\
$\mathrm{C} 29-\mathrm{H} 29 B \cdots \mathrm{O} 1^{\mathrm{i}}$ & 0.99 & 3.52 & $4.4497(17)$ & 156
\end{tabular}

Symmetry codes: (i) $x+1, y, z$; (ii) $-x+2,-y+1,-z+1$; (iii) $-x+1,-y+1,-z+1$; (iv) $-x,-y+2,-z+1$; (v) $x-1, y, z$. 